\title{
Equatorial wave analysis from SABER and ECMWF temperatures
}

\author{
M. Ern ${ }^{1}$, P. Preusse ${ }^{1}$, M. Krebsbach ${ }^{1, *}$, M. G. Mlynczak ${ }^{2}$, and J. M. Russell III ${ }^{3}$ \\ ${ }^{1}$ Institute of Chemistry and Dynamics of the Geosphere (ICG-1), Forschungszentrum Juelich, Juelich, Germany \\ ${ }^{2}$ Atmospheric Sciences Division, NASA Langley Research Center, Hampton, VA, USA \\ ${ }^{3}$ Center for Atmospheric Sciences, Hampton University, Hampton, VA, USA \\ *now at: Department of Physics, University of Wuppertal, Wuppertal, Germany
}

Received: 19 July 2007 - Published in Atmos. Chem. Phys. Discuss.: 8 August 2007

Revised: 1 November 2007 - Accepted: 20 January 2008 - Published: 21 February 2008

\begin{abstract}
Equatorial planetary scale wave modes such as Kelvin waves or Rossby-gravity waves are excited by convective processes in the troposphere. In this paper an analysis for these and other equatorial wave modes is carried out with special focus on the stratosphere using temperature data from the SABER satellite instrument as well as ECMWF temperatures. Space-time spectra of symmetric and antisymmetric spectral power are derived to separate the different equatorial wave types and the contribution of gravity waves is determined from the spectral background of the space-time spectra.

Both gravity waves and equatorial planetary scale wave modes are main drivers of the quasi-biennial oscillation (QBO) in the stratosphere. Temperature variances attributed to the different wave types are calculated for the period from February 2002 until March 2006 and compared to previous findings. A comparison between SABER and ECMWF wave analyses shows that in the lower stratosphere SABER and ECMWF spectra and temperature variances agree remarkably well while in the upper stratosphere ECMWF tends to overestimate Kelvin wave components. Gravity wave variances are partly reproduced by ECMWF but have a significant low-bias. For the examples of a QBO westerly phase (October-December 2004) and a QBO easterly phase (November/December 2005, period of the SCOUT-O3 tropical aircraft campaign in Darwin/Australia) in the lower stratosphere we find qualitatively good agreement between SABER and ECMWF in the longitude-time distribution of Kelvin, Rossby $(n=1)$, and Rossby-gravity waves.
\end{abstract}

Correspondence to: $\mathrm{M}$. Ern

(m.ern@fz-juelich.de)

\section{Introduction}

Equatorial wave modes, such as, for example, Kelvin waves or Rossby-gravity waves, are forced in the tropical troposphere by convective processes (e.g., Pires et al., 1997; Straub and Kiladis, 2003; Lindzen, 2003; Randel and Wu, 2005). As a consequence in the troposphere there are significant contributions of convectively coupled equatorial waves, directly linked to the convective systems acting as wave sources (e.g., Wheeler and Kiladis, 1999; Straub and Kiladis, 2003; Cho et al., 2004).

Equatorial waves also propagate vertically into the stratosphere. Equatorial waves observed in the stratosphere are dominated by "free" wave modes, which are excited by deep convection in the troposphere but not longer linked with the space-time patterns of the convective forcing (Randel and $\mathrm{Wu}, 2005)$.

Together with a broad spectrum of gravity waves equatorial waves are the main drivers of the quasi-biennial oscillation (QBO) in the stratosphere (Hitchman and Leovy, 1988; Dunkerton, 1997; Baldwin et al., 2001). As a result of the interaction with the QBO winds tropical wave activity itself shows modulations due to the QBO.

The QBO is relevant for the stability of the subtropical mixing barrier of the so-called "tropical pipe", which is more stable during QBO easterly phases. Therefore equatorial waves play an indirect but important role for the modulation of mixing processes between the troposphere and the stratosphere by meridional transports (Shuckburgh et al., 2001). Also many other processes in atmospheric chemistry and dynamics in the stratosphere and mesosphere (even at high latitudes) are modulated or influenced by the QBO, showing the importance of the driving equatorial wave modes (Baldwin et al., 2001). Another important effect directly connected with tropical wave activity is tropical upwelling: Wave drag, tropical and subtropical, is an important mechanism for driving

Published by Copernicus Publications on behalf of the European Geosciences Union. 
the observed annual mean tropical upwelling in the lower stratosphere (Semeniuk and Shepherd, 2001).

A theoretical description of planetary scale equatorial wave modes was first given by Matsuno (1966) who derived the properties of the different wave types from solutions of the shallow-water model on an equatorial beta plane with the Coriolis parameter:

$f=\beta y$

where $y$ is the meridional distance from the equator and $\beta$ is the gradient of the Coriolis parameter at the equator. This leads to the following dispersion relation:

$\frac{\sqrt{g h_{e}}}{\beta}\left(\frac{\hat{\omega}^{2}}{g h_{e}}-k^{2}-\frac{k \beta}{\hat{\omega}}\right)=2 n+1, \quad n=0,1,2, \ldots$

with $\hat{\omega}$ the intrinsic frequency of the wave, $k$ the zonal wavenumber, $g$ the gravity acceleration, $n$ the order of the solution, and $h_{e}$ the so-called equivalent depth. The wave modes described by Eq. (2) are trapped near the equator because the Coriolis parameter $f$ changes its sign at the equator.

The equivalent depth is connected with the vertical wavenumber $m$ as given in Eq. (3) (e.g., Wu et al., 2000; Lindzen, 2003):

$m^{2}=\left(\frac{N^{2}}{g h_{e}}-\frac{1}{4 H^{2}}\right)$

with $N$ the buoyancy frequency, and $H$ the pressure scale height.

Equatorial waves can be divided into eastward and westward traveling waves, as well as into wave modes symmetric and antisymmetric with respect to the equator.

In the following the most important wave modes are introduced. For atmospheric temperature the most prominent eastward traveling symmetric wave modes are Kelvin waves (e.g., Tindall et al., 2006a) and, with much smaller amplitude, eastward traveling inertia-gravity waves with $n=1$. Eastward traveling antisymmetric modes are inertia-gravity waves with $n=0$ and $n=2$. Westward traveling symmetric modes are equatorial Rossby waves with $n=1$ and inertiagravity waves with $n=1$, whereas westward traveling antisymmetric wave modes are equatorial Rossby waves and inertia-gravity waves, both with $n=2$, and Rossby-gravity waves.

Figure 1 shows the spectral ranges between the lines for equivalent depths of $h_{e}=8$ and $90 \mathrm{~m}$ under the assumption of zero background wind in the horizontal wavenumber/frequency domain.

These spectral bands are typical for the abovementioned wave modes in the troposphere (Wheeler and Kiladis, 1999). Equivalent depths of 8 and $90 \mathrm{~m}$ correspond to vertical wavelengths between about 2.8 and $9.4 \mathrm{~km}$ in the stratosphere and due to the lower buoyancy frequency $N$ to about 5.6 and $19 \mathrm{~km}$ in the troposphere (see Eq. 3).
For the convectively coupled equatorial waves which are mainly observed in the troposphere (e.g., Wheeler and $\mathrm{Ki}$ ladis (1999)) the nature of convective coupling determines the phase speed and therefore the vertical scale of the waves. Different from this, for the "free" wave modes mainly observed in the stratosphere the tropospheric vertical wavelength values (like the ones given above) can be attributed to the vertical scale of the convective systems acting as source (e.g., Chang, 1976; Fulton and Schubert, 1985; Salby and Garcia, 1987) which is in good agreement with the vertical scale of the heating. Typical vertical profiles of thermal forcing in convective systems have a broad maximum over about 2-8 km in the troposphere (e.g., Chang, 1976; Fulton and Schubert, 1985; Johnson and Ciesielski, 2000). The vertical scales of gravity waves excited by convection are determined by the vertical scale of the heating in a similar way (Alexander et al., 1995).

It should be noted that the frequencies $\omega$ that are observed by satellite instruments and most other observing systems are ground based (Eulerian) frequencies and intrinsic wave frequencies $\hat{\omega}$ will be Doppler shifted in case of non-zero background wind according to:

$\hat{\omega}=\omega-k \bar{u}$

with $k$ the horizontal wavenumber and $\bar{u}$ the background wind. This means that spectral features can shift considerably if plotted against intrinsic frequency instead of ground based frequency. Also the vertical wavelength of the waves considered will be Doppler shifted. For example, a Kelvin wave having $10 \mathrm{~km}$ vertical wavelength for zero background wind will have about $20 \mathrm{~km}$ vertical wavelength if the background wind changes to $-30 \mathrm{~m} / \mathrm{s}$.

Doppler shifting of intrinsic frequencies and vertical wavelengths is not considered throughout the whole paper since the ground based frequency $\omega$ of a wave is "defined" at the wave source level $z_{\text {source }}$ by the wave excitation process as well as the background wind at the source level as follows:

$\omega=\hat{\omega}\left(z_{\text {source }}\right)+k \bar{u}\left(z_{\text {source }}\right)$

and (assuming slowly varying background wind fields) the ground based frequency does not change with altitude - even though the background wind $\bar{u}$ will change with altitude (e.g., Olbers (1981); Marks and Eckermann (1995); Moulin and Flór (2005)). This means: If a wave is launched in the troposphere and propagates through the stratosphere, the "location" of the wave in the zonal-wavenumber/ground-based frequency domain will always be the same, i.e., independent of altitude. Only the intrinsic frequencies (and vertical wavelengths) will change with the background wind and consequently also the lines of fixed equivalent depth will be shifted in a ground based frequency/horizontal wavenumber diagram when the background wind changes.

On the other hand the waves contained at one altitude in one of the frequency bands defined in Fig. 1 will not "leave" 

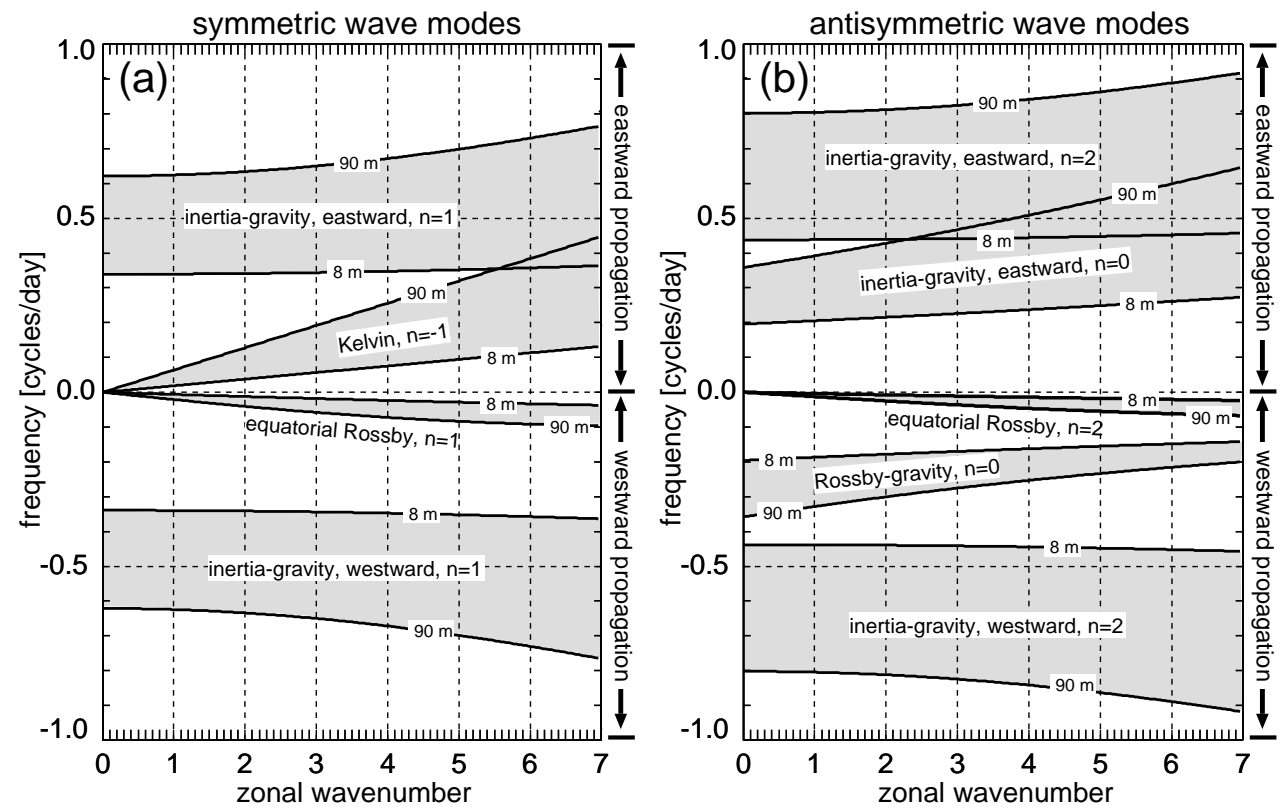

Fig. 1. Spectral ranges between equivalent depth $h_{e}=8 \mathrm{~m}$ and $h_{e}=90 \mathrm{~m}$ for the most relevant equatorial wave modes in the horizontal wavenumber/frequency domain.

this band if the background wind is different at another altitude. As a consequence, if we discuss the properties of the waves contained in one of the wave bands, for example, in an altitude-time cross-section (see Sect. 3) it is the properties of always the same "ensemble" of waves over the whole altitude range and Doppler shifting affects only the amplitudes by, e.g., amplitude modulation, changing the saturation amplitude and critical level filtering (in the latter case the wave is completely obliterated).

It should be noted that because the ground based frequency of a wave remains unchanged the equivalent depth (and vertical wavelength) will be different if the background wind changes and, in particular, the lines of constant equivalent depth for zero background wind drawn in Fig. 1 (or in the space-time spectra shown in Sect. 2) do not indicate the equivalent depth valid for a given spectral feature in the ground based frequency/horizontal wavenumber diagram in case of non-zero background wind. The "real" equivalent depth (or vertical wavelength) of a wave can only be calculated taking into account the Doppler shift caused by the background wind.

Although changes of the background wind are not relevant for the ground based frequencies the Doppler effect can play an important role for the determination of momentum fluxes, which is an important issue to quantify the effect of the analyzed waves on the QBO. Momentum flux cannot be determined from the temperature spectra (temperature variances) presented in our paper alone. Additional information like vertical wavelengths or spectra of wind perturbations would be required. However, this is beyond the scope of this paper.
The phenomenon of Kelvin waves in the oceans is well known for a long time. In the stratosphere first evidence for Kelvin waves was found by Wallace and Kousky (1968) and Rossby-gravity waves were first detected by Yanai and Maruyama (1966) (therefore Rossby-gravity waves sometimes are called Yanai-waves).

Since then numerous studies about equatorial waves have been carried out based on radiosonde data, e.g., Angell et al. (1973); Sato et al. (1994), as well as satellite data providing a global view of the atmosphere. Some examples using stratospheric satellite data are, e.g., Salby et al. (1984); Randel et al. (1990); Randel and Gille (1991); Bergman and Salby (1994); Canziani et al. (1994); Srikanth and Ortland (1998); Tsai et al. (2004); Randel and Wu (2005); Ratnam et al. (2006).

However, all these studies had one or several of the following shortcomings: the data used had only poor spatial or temporal resolution, limited altitude coverage, or the data sets were too short. One of the consequences is that mostly only Kelvin waves and no other wave modes were investigated. Also many analyses focus on zonal wavenumbers 12 , neglecting the higher wavenumbers also important for the dynamics of the QBO.

This has been shown in an analysis by Tindall et al. (2006a,b) in the tropopause region using ERA-15 temperature and wind data: Higher zonal wavenumbers 4-7 also contribute significantly to the momentum flux of the waves. Since the momentum transfer of the different wave types is one of the key drivers of the dynamics of the QBO these high wavenumbers cannot be neglected. 
A comprehensive study in the troposphere was made by Wheeler and Kiladis (1999) using OLR data. In a followup investigation a similar analysis was made by Cho et al. (2004) using TRMM rainfall data. These analyses discuss the largest scale, lowest mode convectively coupled waves which account for only a relatively small fraction of the free waves launched by "background" convection not organized into such waves.

In the stratosphere, different from the troposphere, in particular the Kelvin waves observed (but also other equatorial wave types) cover a larger range of periods. Therefore, for example, the stratospheric Kelvin waves can be classified in ultraslow (periods 25-30 days, Canziani, 1999), slow (periods 10-20 days, Shiotani et al., 1997), fast (periods 610 days, Hitchman and Leovy, 1988), and ultrafast waves (periods 3-4 days, Salby et al., 1984; Lieberman and Riggin, 1997; Garcia et al., 2005). The waves with lower phase speeds $\hat{\omega} / k$ (longer periods for a given zonal wave number) will not propagate to higher altitudes because they will encounter critical level filtering and wave dissipation, for example, by wave breaking or radiative relaxation (e.g., Holton and Lindzen (1972)). In this way the waves interact with the QBO and drive the wind reversal in the stratosphere. On the other hand very high-frequency (short-period) waves are able to penetrate the atmosphere up to the thermosphere. This is why we would expect that the higher up in the atmosphere the more important are the waves with the higher phase speeds and the longer vertical wavelengths and higher equivalent depths (e.g., Salby et al., 2007; Takahashi et al., 2007).

Although a lot of detailed work about equatorial wave modes has been carried out in the stratosphere (see above) there is still a lack of systematic climatological investigations of the different equatorial wave modes based on stratospheric satellite data sets spanning several years.

SABER data have a large potential for the analysis of equatorial waves. A first analysis over a short time period of SABER data with focus on the mesosphere and lower thermosphere has already been carried out by Garcia et al. (2005). Now, having over 4 years of high-quality temperature data from the SABER instrument spanning from the tropopause region to above $100 \mathrm{~km}$ with a good resolution of about $2 \mathrm{~km}$ vertically and covering zonal wavenumbers of up to about 6-7 (owing to the orbit parameters of the TIMED satellite) we have the opportunity to carry out a more comprehensive analysis also in the stratosphere.

\section{Space-time spectral analysis method for equatorial wave modes in SABER and ECMWF data}

\subsection{Analysis method}

To separate symmetric from antisymmetric wave modes often a spectral analysis is carried out dividing the spectral power into its symmetric and antisymmetric parts with respect to the equator (e.g., Wheeler and Kiladis, 1999).

Every data field $\Psi(\lambda, \Phi, t)$ can be written as sum of its symmetric $\Psi_{\text {symm }}(\lambda, \Phi, t)$ and its anti-symmetric $\Psi_{\text {anti }}(\lambda, \Phi, t)$ parts ( $\lambda$ : longitude, $\Phi$ : latitude, $t$ : time):

$$
\begin{aligned}
\Psi(\lambda, \Phi, t)= & \frac{1}{2}(\Psi(\lambda, \Phi, t)+\Psi(\lambda,-\Phi, t)) \\
& +\frac{1}{2}(\Psi(\lambda, \Phi, t)-\Psi(\lambda,-\Phi, t)) \\
=: & \Psi_{\text {symm }}(\lambda, \Phi, t)+\Psi_{\text {anti }}(\lambda, \Phi, t)
\end{aligned}
$$

And we also obtain symmetric $\hat{\Psi}_{\text {symm }}(k, \omega ; \Phi)$ and antisymmetric $\hat{\Psi}_{\text {anti }}(k, \omega ; \Phi)$ spectral power after Fourier transform in longitude and time ( $k$ : zonal wavenumber, $\omega$ : wave frequency). The total spectral power contained in $\Psi(\lambda, \Phi, t)$ is:

$$
\begin{aligned}
\hat{\Psi}(k, \omega ; \Phi)= & \sum_{\lambda, t} \Psi(\lambda, \Phi, t) \exp (i(k \lambda+\omega t)) \\
= & \sum_{\lambda, t}\left(\Psi_{\text {symm }}(\lambda, \Phi, t)+\Psi_{\text {anti }}(\lambda, \Phi, t)\right) \\
& \times \exp (i(k \lambda+\omega t)) \\
= & \hat{\Psi}_{\text {symm }}(k, \omega ; \Phi)+\hat{\Psi}_{\text {anti }}(k, \omega ; \Phi)
\end{aligned}
$$

Since equatorial wave modes are expected to be either symmetric or antisymmetric with respect to the equator often the symmetric and antisymmetric spectral power are treated independently and each averaged over a latitude band (e.g., $15 \mathrm{~S}-15 \mathrm{~N}$ ) after first calculating the power at each individual latitude to increase the signal to noise ratio.

In our case the data analyzed in this way are residual temperatures from the SABER instrument and European Centre for Medium-Range Weather Forecasts (ECMWF) analyses. Some information about the ECMWF model and the recent changes in the ECMWF data assimilation system are given, for example, in Jung and Leutbecher (2007).

We use a windowed Fourier analysis based on nonoverlapping 31-day time windows. The choice of this window length is some kind of compromise between the expected temporal variations of the data and at the same time still sufficient frequency resolution. For example, in the upper stratosphere effects due to the semi-annual oscillation (SAO) can be found, so the window length has to be well below 3 months not to smooth out the temporal variations. On the other hand ultraslow Kelvin waves can have periods of about 30 days which can still be represented with the window length used. In addition, also the stationarity of the wave modes we expect to find plays an important role as we expect to find also shorter period waves than in the troposphere.

It should however be noted that this compromise of a 31day time-window implicates some limitations to our method: Some spectral leakage is expected if there is a mismatch between the ground based frequency of a wave and the spectral grid points used in the spectral analysis. Especially cases with periods close to 31 days (maybe even longer than the 
time windows used) could be problematic. In such cases there will be an underestimation of the 31-day component and some contamination of neighbored frequencies. On the other hand such long periods are only prominent at the lowermost altitudes and the main findings presented in this paper are not affected by this uncertainty.

\subsection{Analysis of SABER data}

The SABER instrument onboard the TIMED satellite measures temperatures and several trace gases from the tropopause region to above $100 \mathrm{~km}$ (e.g., Mlynczak, 1997; Russell et al., 1999; Yee et al., 2003). In this paper we will analyze temperature residuals from the zonal mean for version 1.06 SABER temperature data.

With the TIMED orbit cycle of about $1.7 \mathrm{~h}$ (i.e., 14 orbits/day) SABER data can resolve zonal wavenumbers up to 6-7 and frequencies up to about 1 cycle/day. Further details about the asynoptic sampling geometry of low Earth orbiting satellites can be found in, e.g., Hayashi (1980); Salby (1982a,b); Wu et al. (1995). Due to the asynoptic sampling simple fast Fourier transform (FFT) cannot be applied to the satellite data. Instead, we use a least-squares method similar to the approach described by Wu et al. (1995) which has already been used by Smith et al. (2002) to estimate the space-time Fourier coefficients for an analysis of equatorial wave signatures in CRISTA temperature data. This kind of approach can also cope with data gaps as well as irregular satellite sampling pattern (Wu et al., 1995), which is important because the TIMED satellite performs yaw maneuvers every 60 days due to solar angle restrictions.

We obtain residual SABER temperatures by subtracting the zonal wavenumber zero of a Kalman filter analysis, giving a temporally evolving estimate for the zonal mean on a daily basis. The data set we use ranges from February 2002 until March 2006. The equatorial wave analysis of SABER temperatures is carried out in $4^{\circ}$ latitude bins centered at the equator and $\pm 4, \pm 8, \pm 12, \ldots$ degrees latitude. The analysis covers altitudes in $1-\mathrm{km}$ steps, starting at $20 \mathrm{~km}$ as the lowermost altitude to avoid altitudes with increased noise due to cloud-contamination of the observations. The uppermost analysis altitude was above $100 \mathrm{~km}$, however, we will focus on the stratospheric analyses in the altitude range $20-50 \mathrm{~km}$ and a comparison to results from ECMWF in this paper.

Figure $2 \mathrm{a}$ and $\mathrm{b}$ show space-time spectra of symmetric and antisymmetric squared spectral amplitudes in $\mathrm{K}^{2} /$ wavenumber/cpd (i.e., two times power spectral density) at $21 \mathrm{~km}$ altitude averaged over the whole period analyzed (Feb 2002 until March 2006) and over latitudes 14 S-14 N (i.e., the analyses for the latitude bins $0, \pm 4, \pm 8, \pm 12$ degrees are averaged) from SABER residual temperatures.

Also given are the lines for equivalent depths of 8,90 and $2000 \mathrm{~m}$ under the assumption of zero background wind, corresponding to vertical wavelengths of about 3,9 and $50 \mathrm{~km}$ in the stratosphere (also under the assumption of zero back- ground wind). The spectral regions defined by these lines will be used for integration of the total spectral contributions of the different wave types in Sect. 3. Please note that in Fig. 2 the color scales are different for symmetric and antisymmetric spectra.

It first should be mentioned that in stratospheric temperatures we obviously do not have a red noise-like background spectrum dominating over most of the spectral signatures of equatorial waves like in Wheeler and Kiladis (1999) or Cho et al. (2004), who analyzed tropospheric OLR and precipitation data. In our analysis of stratospheric temperatures the spectral peaks of Kelvin waves in symmetric spectral power and of Rossby-gravity waves in antisymmetric power are the most prominent spectral features (beneath equatorial Rossby waves at very low frequencies and diurnal variations at $\pm 1 \mathrm{cpd}$ ).

In Fig. 2 we can see that already in the lower stratosphere spectral contributions are somewhat shifted towards higher equivalent depths with respect to the tropospheric observations by Wheeler and Kiladis (1999) or Cho et al. (2004). There are also contributions of the different wave types outside the $8-90$ m equivalent depth wave bands and a large portion of the faster stratospheric signals are likely independent of the tropospheric waves studied by Wheeler and Kiladis (1999).

Both the spectra shown in Fig. 2 and the tropospheric observations by Wheeler and Kiladis (1999) or Cho et al. (2004) are ground-based frequency/zonal wavenumber spectra. Since the ground-based frequency of a wave does not change when the wave encounters vertical wind shear, the shift of the spectral contributions with respect to the troposphere indicates that at low (ground-based) frequencies there is a real loss of spectral power (Doppler shifting of the waves would have no effect on the ground-based frequencies observed).

At the same time the occurrence of higher ground-based frequencies (higher equivalent depths) could be an indication for processes involving longer vertical scales in the troposphere that become important only in the stratosphere and become visible due to amplitude growth with altitude but are too small effects in the troposphere to be observed.

The loss of spectral power at low ground-based frequencies (lower equivalent depths) can have different reasons, such as critical level filtering and wave dissipation due to, e.g., wave breaking or radiative relaxation. Another reason could be wave amplitude modulations of waves propagating conservatively caused by changes in the background wind.

The change in the static stability will lead to a significant increase of wave amplitudes during the transition of a wave from the troposphere into the stratosphere because the buoyancy frequency $N$ increases by a factor of about two. In the whole stratosphere, however, this should be a minor effect because $N$ is about constant in the stratosphere.

For the example of Kelvin waves, which satisfy the same dispersion relation as gravity waves, we can make use of 

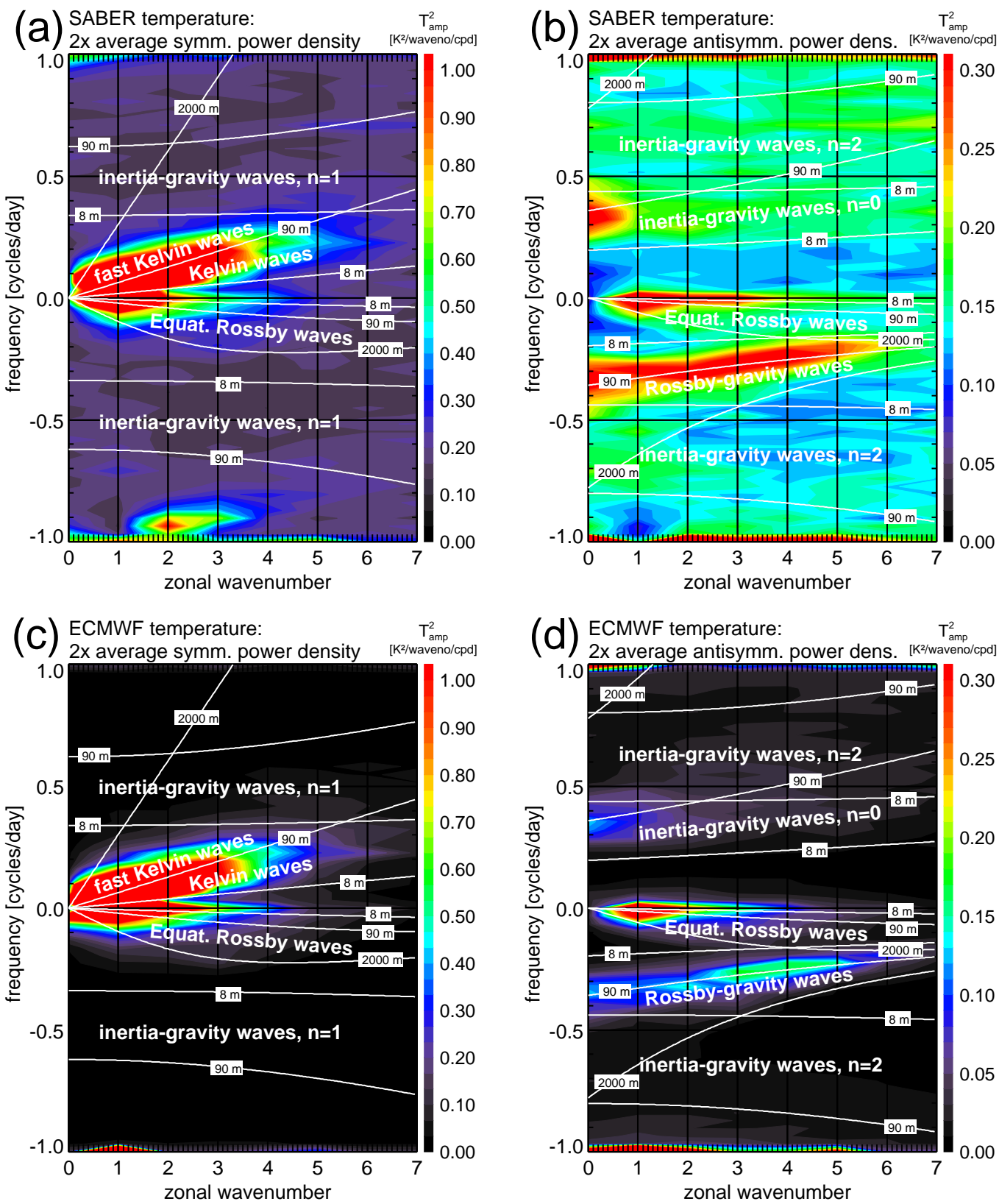

Fig. 2. Space-time spectra of SABER temperature symmetric (a) and antisymmetric (b) squared spectral amplitudes at $21 \mathrm{~km}$ altitude, averaged over the whole period analyzed (Feb 2002 until March 2006) and over latitudes 14 S-14 N (i.e., the analyses for the latitude bins $0, \pm 4, \pm 8, \pm 12$ degrees are averaged). Also shown: ECMWF temperature symmetric (c) and antisymmetric (d) squared spectral amplitudes at $21 \mathrm{~km}$ altitude, averaged over the whole period analyzed (Feb 2002 until March 2006) and over latitudes 15 S-15 N. For comparison the lines for equivalent depths of 8,90 , and $2000 \mathrm{~m}$ are given for the different wave modes.

Eq. (55) in Fritts and Alexander (2003), which was originally derived for gravity waves. Following Eq. (55) in Fritts and Alexander (2003) for the transition from troposphere into stratosphere a change of temperature variances according to:

$\left(\overline{T^{\prime 2}}\right)_{\text {strato }} /\left(\overline{T^{\prime 2}}\right)_{\text {tropo }} \approx N_{\text {strato }}^{3} / N_{\text {tropo }}^{3}$

would be expected with $\left(\overline{T^{\prime 2}}\right)_{\text {tropo }}$ the tropospheric and
$\left(\overline{T^{\prime 2}}\right)_{\text {strato }}$ the stratospheric value. In this equation changes in temperature and atmospheric density are neglected. Since in the stratosphere the buoyancy frequency $N_{\text {strato }}$ is about twice the value in the troposphere $N_{\text {tropo }}$ this would be an increase in variances (and power spectral densities) of almost an order of magnitude. 

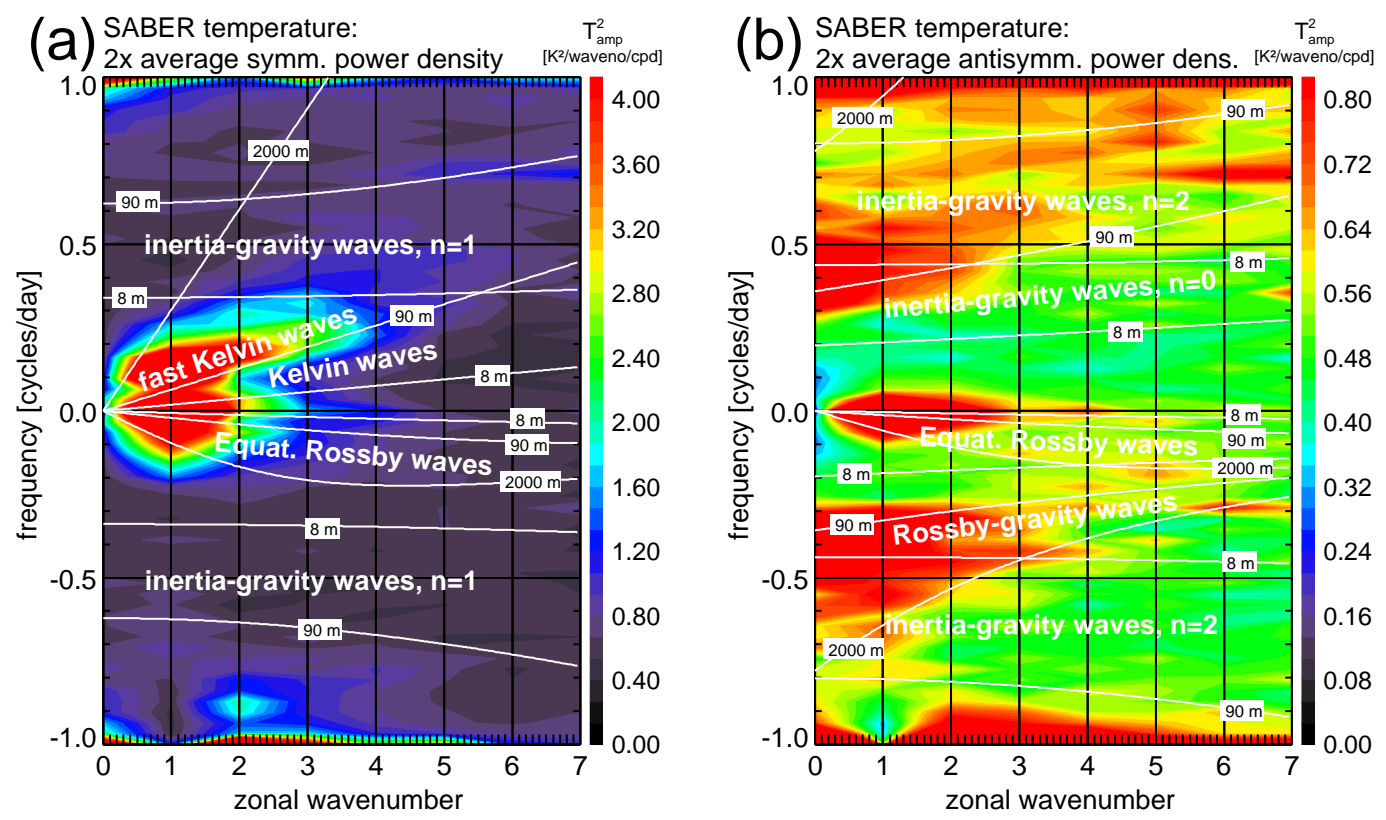

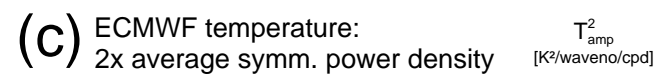
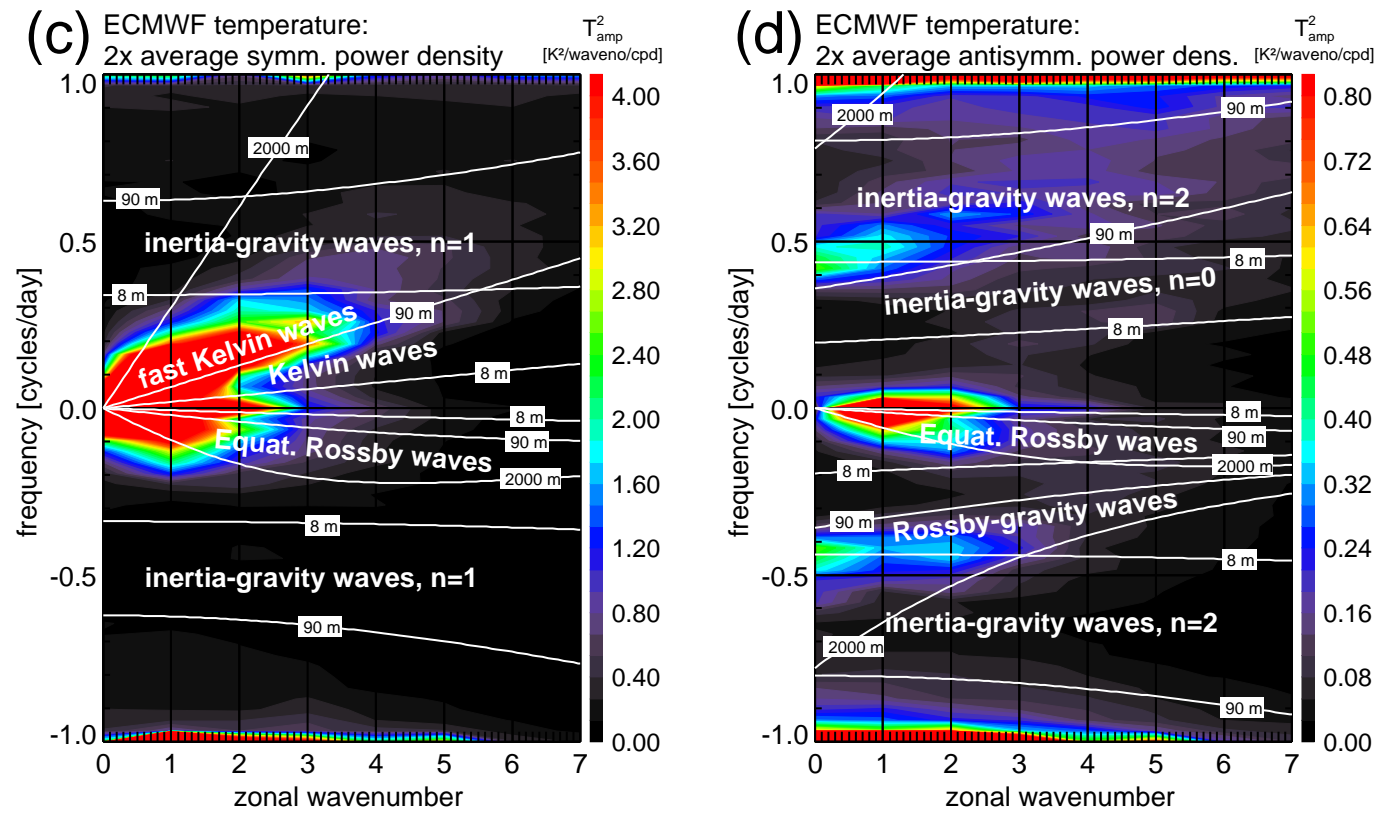

Fig. 3. Space-time spectra of SABER temperature symmetric (a) and antisymmetric (b) squared spectral amplitudes at $41 \mathrm{~km}$ altitude, averaged over the whole period analyzed (Feb 2002 until March 2006) and over latitudes 14 S-14 N (i.e., the analyses for the latitude bins 0, $\pm 4, \pm 8, \pm 12$ degrees are averaged). Also shown: ECMWF temperature symmetric (c) and antisymmetric (d) squared spectral amplitudes at $41 \mathrm{~km}$ altitude, averaged over the whole period analyzed (Feb 2002 until March 2006) and over latitudes $15 \mathrm{~S}-15 \mathrm{~N}$. For comparison the lines for equivalent depths of 8,90 , and $2000 \mathrm{~m}$ are given for the different wave modes.

Both fast and slow waves will encounter amplitude amplification due to this change in $N$ as well as due to the decrease of atmospheric density with altitude. However for the slow waves obviously critical level filtering and wave dissipation or amplitude modulation due to changes in the background wind are more important and a relative shift of the spectral signatures is observed.
The fact that the values shown in Figs. 2 and 3 are squared spectral amplitudes (in units of $\mathrm{K}^{2} /$ wavenumber/cpd) means that we can apply Parseval's theorem to calculate variances from the spectral values given by integrating the spectral values over a given area in the wavenumber/frequency domain. According to Parseval's theorem the spectral density integrated over the whole spectral domain is equal to the overall 
variance of the data set analyzed. In our case it has to be kept in mind that the squared spectral amplitudes we use are two times power spectral density and the integration result has to be divided by two to obtain the variance. In addition, symmetric and antisymmetric spectra have to be treated separately.

The calculation of variances will now be demonstrated in an example: Beneath the abovementioned spectral peaks we find some kind of continuous spectral background which is about constant in the wavenumber/frequency-domain. For both symmetric and antisymmetric spectra it is about $0.15 \mathrm{~K}^{2} /$ wavenumber/cpd. By integrating over the whole wavenumber/frequency-domain in both symmetric and antisymmetric spectra, which have to be treated separately, this would be equal to a contribution of about $2 \mathrm{~K}^{2}$ of SABER temperature variances. The contributions from symmetric and antisymmetric backgrounds (both $0.15 \mathrm{~K}^{2} /$ waveno./cpd) are added in the following way:

$\left((0.15+0.15) \mathrm{K}^{2} /\right.$ waveno./cpd $\times 7$ wavenos. $\times 2$ cpd $) / 2 \approx 2 \mathrm{~K}^{2}$

Of course, this background is partly composed of measurement noise, interpolation errors, spectral leakage and aliasing, as well as contributions from inertia-gravity waves with $n>0$. But the main contribution are probably localized gravity waves which are not resolved by the analysis method, playing also an important role in equatorial atmospheric dynamics (see Sect. 1).

The contribution of inertia-gravity waves with $n>0$ cannot be distinguished from this background. Therefore inertiagravity waves with $n>0$ will not be subject of this paper.

\subsection{Analysis of ECMWF data}

The ECMWF data set we use has a spatial resolution of 1 deg longitude times 1 deg latitude. Data are available daily for 00:00, 06:00, 12:00, 18:00 GMT on 28 pressure levels between 1013.25 and 0.1 mbar. For comparison with the SABER data given on geometric altitudes the ECMWF pressure levels are converted to pressure-altitude coordinates $z_{i}$ using a constant scale height of $7 \mathrm{~km}$ :

$z_{i}=7 \mathrm{~km} * \ln \left(p_{0} / p\right)$

with $\mathrm{p}_{0}=1013.25 \mathrm{hPa}$. Because we are mainly interested in a comparison between SABER and ECMWF we use only ECMWF data from the same period where SABER data are available (Feb 2002 until March 2006) and focus on stratospheric altitudes.

Although SABER data are not assimilated in ECMWF some agreement between SABER and the ECMWF operational analyses is expected because other satellite data (TOVS/ATOVS radiances) are used for data assimilation in the stratosphere. The TOVS/ATOVS radiances are sounded in nadir viewing geometry with broad vertical weighting functions in both troposphere and stratosphere (see $\mathrm{Li}$ et al.,
2000). Therefore it would be expected that long vertical wavelength waves (higher equivalent depths) are better represented in ECMWF than short vertical wavelength waves (lower equivalent depths).

The ECMWF data offer a much better space-time resolution than the SABER data. Zonal wavenumbers as high as 180 and frequencies up to 2 cycles/day (cpd) can be resolved. For the current analysis such high resolution is not required. We use the full latitudinal resolution of $1 \mathrm{deg}$, but only a reduced data set with 9 deg longitude resolution. To make sure that the variance of the data is not affected we simply omit ECMWF grid points in our analysis. This reduced data set covers zonal wavenumbers up to 20 and frequencies up to 2 cycles/day. Full resolution control runs show that almost no information is lost because most equatorial wave activity is at zonal wavenumbers lower than 15 .

Residual ECMWF temperatures are obtained using a detrending method different from the one used for the SABER data, which was based on daily zonal mean values (see Sect. 2.2). Instead, for ECMWF we calculate linear fits of the data in each of the 31-day time windows for every pressure level and latitude. These fits are subtracted from the data time window by time window to remove mean values as well as temporal trends. Since the time windows used are relatively short (31 days) there are no contaminations by the annual cycle and the results of the analyses show that the method of detrending the data has almost no effect. Another difference to the SABER analysis is that, owing to the fact that ECMWF data are given on a regular grid in space and time, the FFT can be used to determine the space-time Fourier coefficients.

\subsection{Comparison of the SABER and ECMWF analyses}

Like the average SABER spectra shown in Fig. 2a and b, Fig. $2 \mathrm{c}$ and d show space-time spectra of symmetric and antisymmetric squared spectral amplitudes (two times power spectral density) at $21 \mathrm{~km}$ altitude, averaged over the whole period analyzed (Feb 2002 until March 2006) and over latitudes $15 \mathrm{~S}-15 \mathrm{~N}$ for ECMWF. Please note that the color scales are the same as in Fig. 2a and b but only part of the full spectral range of the ECMWF data set used is shown (see Sect. 2.3).

There are striking similarities in the shape of the spectral peaks of Kelvin, equatorial Rossby, and Rossby-gravity waves. Even the occurrence of fast Kelvin or Rossby-gravity waves outside the $8-90 \mathrm{~m}$ equivalent depth spectral bands can be found in the ECMWF data. Again, it is difficult to separate the contribution of inertia-gravity waves from the background.

The most striking difference between average SABER and ECMWF spectra can be found in the quasi continuous background which is much lower in the ECMWF spectra. Partly this is an effect of the larger spectral region covered by ECMWF so that the total background variance is distributed on a larger number of spectral grid points. Therefore 
a reduced background would be expected in Fig. 2c and d, which show only the part of the spectral domain common with the SABER analysis and not the full spectra. However, the total contribution of the ECMWF spectral background (symmetric and antisymmetric added) is equivalent to temperature variance between about 0.5 and $0.7 \mathrm{~K}^{2}$, which is considerably lower than the SABER value of about $2 \mathrm{~K}^{2}$, indicating that this is not only an effect of the larger spectral domain covered by ECMWF. Smaller parts of this difference can be attributed to interpolation errors or measurement noise present in SABER data but not in ECMWF. The largest contribution, however, is most likely due to the broad spectrum of gravity waves which has been found before in SABER temperature data (e.g., Preusse et al., 2006; Krebsbach and Preusse, 2007) and other limb sounding satellite data sets (e.g., Fetzer and Gille, 1994; Eckermann and Preusse, 1999; Preusse et al., 2000, 2002; Ern et al., 2004, 2005, 2006; Wu et al., 2006) but is obviously underrepresented in the ECMWF data used.

Figure $3 a-d$ shows the average spectra for SABER and ECMWF at $41 \mathrm{~km}$ altitude.

Please note that again the color scales are different for symmetric and antisymmetric spectra. Compared to Fig. 2a and $\mathrm{c}$ the spectral peak attributed to Kelvin waves is shifted towards higher equivalent depths. For comparison the lines for equivalent depths 8,90 and $2000 \mathrm{~m}$ under the assumption of zero background wind are also shown.

This shift probably indicates that part of the Kelvin waves at lower ground based phase speeds (i.e., lower equivalent depths) is absorbed in the lower stratosphere, thereby transferring momentum to the zonal wind system and driving the QBO. On the other hand Kelvin waves with higher equivalent depths, which have higher phase speeds, are not affected and can propagate towards higher altitudes. The effects observed for the other wave modes are similar.

For the differences between the spectra at $21 \mathrm{~km}$ and $41 \mathrm{~km}$ altitude amplitude modulations of waves propagating conservatively caused by changes in the background static stability can be neglected because the buoyancy frequency $N$ is about constant throughout the whole stratosphere. Also amplitude modulations due to the vertical shear of the background wind will in many cases be small compared to the expected increase of wave variances due to the decrease of atmospheric density over three pressure scale heights: An increase of wave variance of about a factor of 20 would be expected.

The quasi continuous background in the SABER spectra is strongly enhanced compared to $21 \mathrm{~km}$ altitude. The temperature variances contributed to this background are about $7 \mathrm{~K}^{2}$. This likely reflects the increase of the amplitudes of gravity waves with altitude. There is also an enhancement in the ECMWF background at $41 \mathrm{~km}$ altitude compared to $21 \mathrm{~km}$. The temperature variance due to this background is about $3-4 \mathrm{~K}^{2}$, again considerably lower than the SABER val-
Table 1. Squared spectral amplitudes of the strongest Kelvin wave components in the four-year average SABER and ECMWF temperature analyses at 21,32 , and $41 \mathrm{~km}$ altitude.

\begin{tabular}{|c|c|c|}
\hline $\begin{array}{l}k: \text { zonal waveno., } \\
T: \text { period [days] }\end{array}$ & $\begin{array}{c}\text { SABER } \\
\text { squared spectral } \\
\text { amplitude } \\
{\left[\mathrm{K}^{2} / \text { waveno/cpd }\right]}\end{array}$ & $\begin{array}{c}\text { ECMWF } \\
\text { squared spectral } \\
\text { amplitude } \\
{\left[\mathrm{K}^{2} / \text { waveno/cpd }\right]}\end{array}$ \\
\hline \multicolumn{3}{|l|}{ altitude: $21 \mathrm{~km}$} \\
\hline$k=1, T=15.50$ & 6.32 & 6.11 \\
\hline$k=1, T=10.33$ & 6.62 & 6.42 \\
\hline$k=2, T=10.33$ & 3.52 & 3.72 \\
\hline$k=2, T=7.75$ & 2.98 & 2.62 \\
\hline$k=2, T=6.20$ & 2.14 & 1.74 \\
\hline \multicolumn{3}{|l|}{ altitude: $32 \mathrm{~km}$} \\
\hline$k=1, T=15.50$ & 5.13 & 6.47 \\
\hline$k=1, T=10.33$ & 8.50 & 11.27 \\
\hline$k=1, T=7.75$ & 4.55 & 4.37 \\
\hline$k=1, T=6.20$ & 2.50 & 2.37 \\
\hline$k=2, T=7.75$ & 3.37 & 3.95 \\
\hline$k=2, T=6.20$ & 3.29 & 3.89 \\
\hline$k=2, T=5.17$ & 2.27 & 2.58 \\
\hline \multicolumn{3}{|l|}{ altitude: $41 \mathrm{~km}$} \\
\hline$k=1, T=15.50$ & 4.57 & 9.21 \\
\hline$k=1, T=10.33$ & 9.20 & 18.14 \\
\hline$k=1, T=7.75$ & 8.13 & 12.02 \\
\hline$k=1, T=6.20$ & 6.92 & 8.74 \\
\hline$k=2, T=7.75$ & 2.41 & 4.75 \\
\hline$k=2, T=6.20$ & 4.82 & 6.12 \\
\hline$k=2, T=5.17$ & 4.24 & 5.73 \\
\hline$k=2, T=4.43$ & 3.01 & 4.13 \\
\hline
\end{tabular}

ues. The contribution due to gravity waves will be discussed in more detail in Sect. 3.2.

The strongest Kelvin wave components in the 4-year average spectra are given in Table 1 for both SABER and ECMWF at the altitudes 21,32 , and $41 \mathrm{~km}$.

We have chosen only the strongest spectral contributions because those should only be little influenced by differences in the spectral background (see also below). At altitudes below about $32 \mathrm{~km}$ the values given in Table 1 for SABER and ECMWF are very similar and mostly do not differ by more than $10-20 \%$. At higher altitudes the strongest Kelvin wave components found in ECMWF are higher than the SABER values on average and can exceed the SABER values by $50 \%$ and more. This can also be seen from Fig. 4 where the average deviation of the 10 strongest 4-year average Kelvin wave components is plotted against the altitude (solid line).

Also given in Fig. 4 is the relative deviation of the total ECMWF Kelvin wave variances from the SABER variances (long dashed line) in the spectral region $k=1-6$ and frequencies between 0.03 and 0.4 cycles/day. ECMWF variances are 


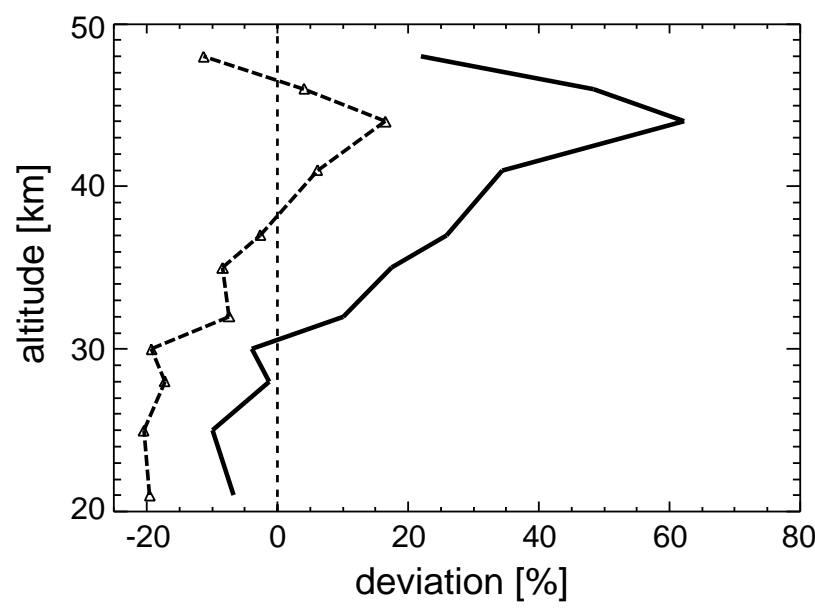

Fig. 4. Altitude profile of the average deviation between the corresponding ECMWF analysis components and the 10 strongest 4year average Kelvin wave components in the SABER analysis (bold solid line). Also given: the relative deviation of the total ECMWF Kelvin wave variances from the SABER variances (long dashed line).

somewhat lower in the lower stratosphere and about the same in the upper stratosphere.

However, it should be noted that the relative deviations shown in Table 1 and Fig. 4 are calculated for squared spectral amplitudes given in $\mathrm{K}^{2} /$ waveno./cpd (see also Figs. 2 and 3 ). Since the relative deviation doubles by squaring the values, relative deviations would be lower by a factor of two if Kelvin wave amplitudes are considered.

This means that not only the relative distribution of the spectra is very similar, but also the absolute values are in good agreement in the lower stratosphere. In the upper stratosphere ECMWF tends to overestimate Kelvin wave components.

\section{Timeseries for the different wave components}

\subsection{Equatorial wave modes}

In the previous section average spectra at $21 \mathrm{~km}$ and $41 \mathrm{~km}$ altitude were shown and good agreement was found between SABER and ECMWF. Now the question arises whether this agreement still holds if we extend the comparison over the whole stratosphere and take into account the temporal evolution of equatorial wave activity. For this analysis we use the same 31-day time windows as in Sect. 2. The temperature variances that will be determined in each time window are attributed to the center days of the time windows.

In a first step we integrate the power spectral density over the spectral bands between 8 and $2000 \mathrm{~m}$ equivalent depth to calculate the "total" temperature variances of the different wave modes. For both SABER and ECMWF we inte- grate only over zonal wavenumbers $1-6$. The choice of 8 and $2000 \mathrm{~m}$ is somewhat arbitrarily and the reason for this spectral range is that most of the contributions of a given wave mode will be contained in this spectral band in the whole altitude range of $20-40 \mathrm{~km}$ (see Figs. 2 and 3). And the choice of an upper limit of $2000 \mathrm{~m}$ equivalent depth, for example, would also include the ultrafast Kelvin waves identified by Salby et al. (1984), having wavenumber 1 and periods of about 3.5 days.

Similar as in Wheeler and Kiladis (1999) we cut the spectral bands used for integration at $0.4 \mathrm{cpd}$ for Kelvin waves and at $0.5 \mathrm{cpd}$ for eastward inertia-gravity waves $(n=0)$ and -0.5 cpd (i.e., only periods longer than about 2 days) for Rossby-gravity waves to avoid too large overlaps with the frequency bands attributed to tides and inertia-gravity waves with higher values of $n>0$.

Figure 5 shows the altitude-time cross-sections obtained for SABER and Fig. 6 for ECMWF.

The values given are temperature variances calculated from the integrated squared spectral amplitudes (two times power spectral densities). Shown are the contributions due to (a) Kelvin waves, (b) inertia-gravity waves $(n=0)$, (c) equatorial Rossby waves $(n=1)$, and (d) Rossby-gravity waves, respectively. The contributions due to inertia-gravity waves $(n>0)$ and equatorial Rossby waves $(n=2)$ are not shown because the amplitudes are rather small compared to the spectral background.

Overplotted in Figs. 5 and 6 are contour lines of the zonal mean zonal wind from ECMWF averaged over the 31-day windows used and all latitudes from $15 \mathrm{~S}-15 \mathrm{~N}$. The contour interval is $10 \mathrm{~m} / \mathrm{s}$. Solid lines indicate eastward, dashed lines westward wind. The zero wind line is highlighted by a boldface solid line.

As expected there is an evident modulation of the temperature variances by the QBO below about $40 \mathrm{~km}$ altitude. Eastward propagating wave modes like Kelvin waves (panels a) or $n=0$ inertia-gravity waves (panels b) show enhanced values during QBO east phases (i.e., phases of westward directed zonal winds) while westward propagating wave modes like $n=1$ equatorial Rossby waves (panels c) or Rossbygravity waves (panels d) are enhanced during QBO west phases (i.e., phases of eastward directed zonal winds).

At altitudes above $40 \mathrm{~km}$ the zonal mean zonal wind is not longer dominated by the QBO pattern of alternating eastward and westward wind with an oscillation period of about 24 months. Instead, the zonal mean zonal wind is more and more dominated by an oscillation with a period of about 6 months. This semiannual oscillation (SAO) of the zonal wind can be found in the upper stratosphere and the mesosphere. From Figs. 5 and 6 we see that above above $40 \mathrm{~km}$ altitude also the temperature variances are dominated by the $\mathrm{SAO}$ and exhibit the same semiannual variation as the zonal wind.

Kelvin waves are the by far dominant wave mode in residual temperatures, reaching temperature variances of about 

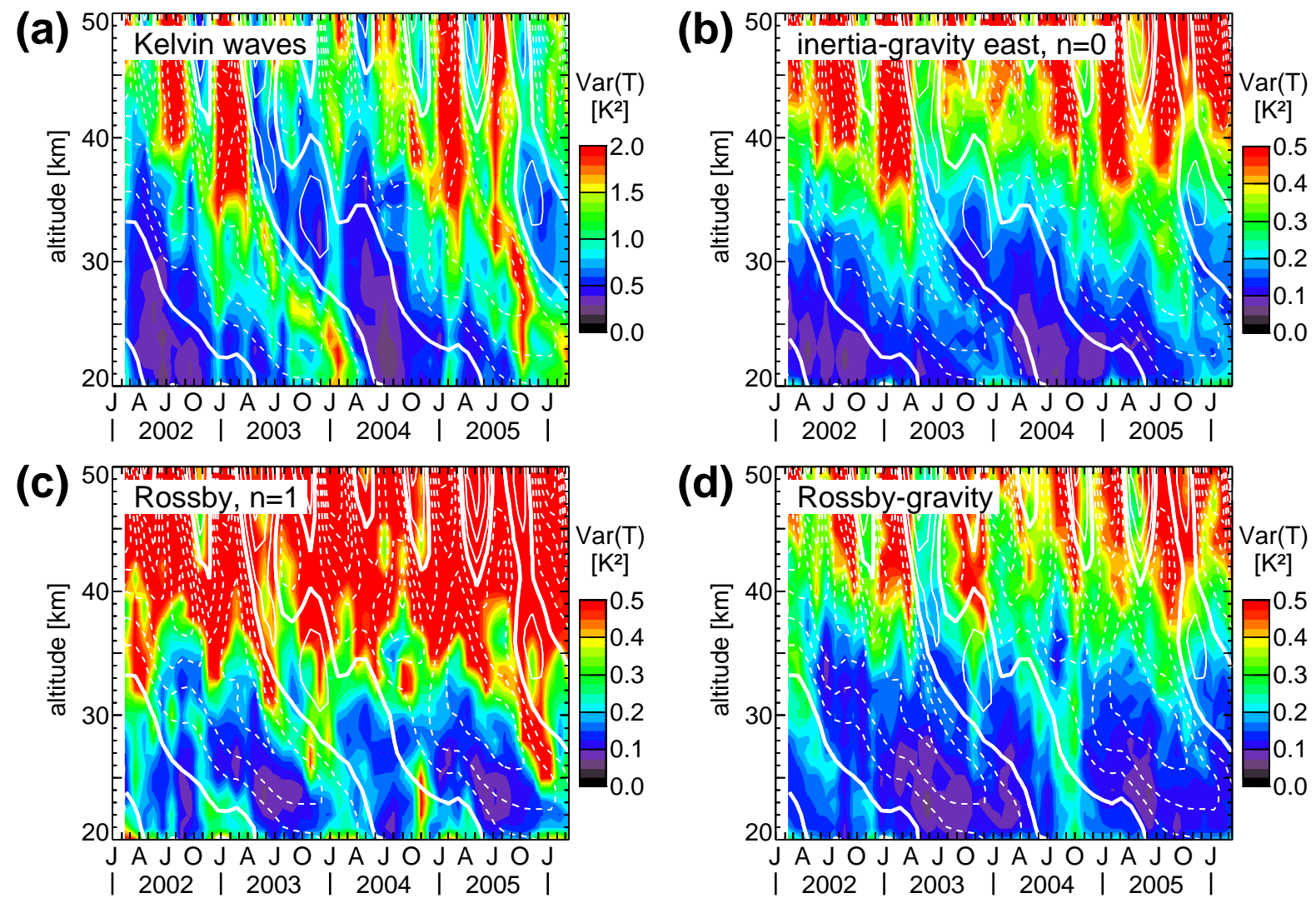

Fig. 5. Altitude-time cross-sections of SABER temperature variances integrated over the wave bands between 8 and $2000 \mathrm{~m}$ equivalent depth for (a) Kelvin waves, (b) inertia-gravity waves $(n=0)$, (c) equatorial Rossby waves $(n=1)$, and (d) Rossby-gravity waves. Overplotted contour lines are zonal mean zonal wind from ECMWF averaged over the latitudes $15 \mathrm{~S}-15 \mathrm{~N}$. Contour interval is $10 \mathrm{~m} / \mathrm{s}$, solid lines indicate eastward, dashed lines westward wind. The zero wind line is highlighted by a boldface solid line.

1.5-2 $\mathrm{K}^{2}$ during QBO east phases. Temperature variances of the other wave modes are small compared to the Kelvin waves. Please note that in Figs. 5 and 6 the color scales are different for Kelvin waves and the other wave modes.

In the following the $8-2000 \mathrm{~m}$ equivalent depth spectral band will be split up into a "slow" spectral band, ranging from 8-90 m equivalent depth (about the same range as used by Wheeler and Kiladis (1999)) and a "fast" spectral band, ranging from 90-2000 $\mathrm{m}$ equivalent depth. We distinguish between the "slow" and the "fast" wave bands because we expect different responses of the "slow" and the "fast" waves to the QBO. "Slow" and "fast" in this context means: slower, respectively, faster (ground based) phase speeds of the waves at a given zonal wavenumber.

Figures 7 and 8 show the same as Figs. 5 and 6 but for the contribution of only lower equivalent depths between 8 and $90 \mathrm{~m}$ in the "slow" wave bands, whereas Figs. 9 and 10 show the contribution of only the higher equivalent depths between 90 and $2000 \mathrm{~m}$ in the "fast" wave bands.

Please note that the color scales in Figs. 7-10 are different from the ones in Figs. 5 and 6.
From Figs. 9a and 10a we can see that for the Kelvin waves obviously the modulation of the spectral power in the "fast" wave band is not as strong as for the "slow" wave band, especially in the middle and upper stratosphere. The QBO modulation of the "slow" wave band equatorial waves observed in Figs. 7 and 8 is consistent with results for long-period Kelvin waves by Wallace and Kousky (1968) and Rossby-gravity waves by Sato et al. (1994). For the Kelvin waves in the "slow" wave band variances vary from about $0.1 \mathrm{~K}^{2}$ to about $1 \mathrm{~K}^{2}$ between maximum and minimum values in the stratosphere. This means there is a contrast of a factor of about 10 caused by QBO modulation. In the SABER "fast" wave band (Fig. 9a) the contrast between maximum and minimum variance, for example, is only a factor of about $2-3$ at $30 \mathrm{~km}$ altitude.

It is also remarkable that the QBO induced maxima of the "slow" wave band are very narrow and occur mainly during periods of eastward shear in the westward wind of the QBO east periods (see also Ratnam et al., 2006). This means that these waves are closely related with the downward phase propagation of the westward background winds of the QBO east phases. Different from this the "fast" band Kelvin waves 

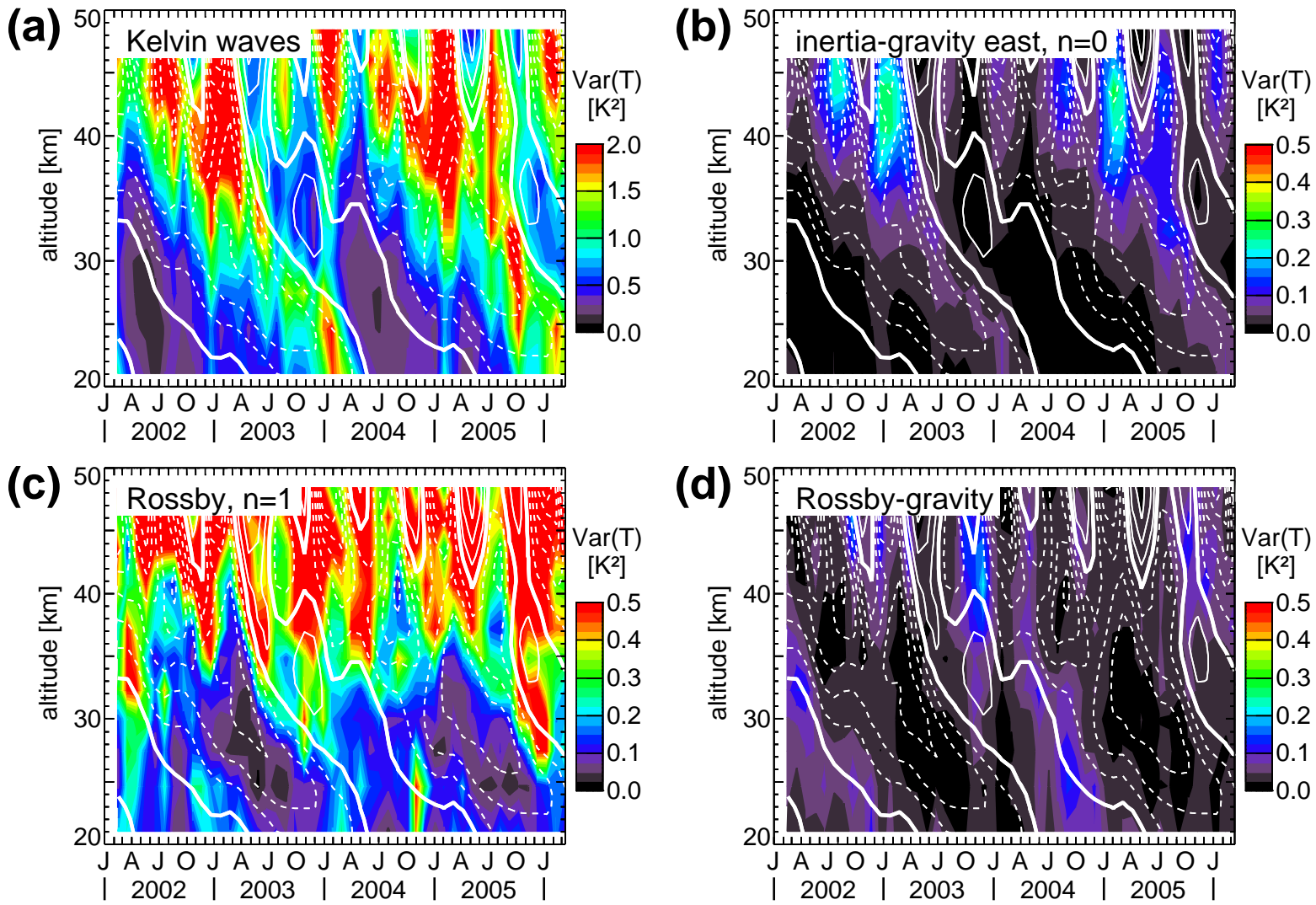

Fig. 6. Altitude-time cross-sections of ECMWF temperature variances integrated over the wave bands between 8 and $2000 \mathrm{~m}$ equivalent depth for (a) Kelvin waves, (b) inertia-gravity waves $(n=0)$, (c) equatorial Rossby waves $(n=1)$, and (d) Rossby-gravity waves. Overplotted contour lines are zonal mean zonal wind from ECMWF averaged over the latitudes $15 \mathrm{~S}-15 \mathrm{~N}$. Contour interval is $10 \mathrm{~m} / \mathrm{s}$, solid lines indicate eastward, dashed lines westward wind. The zero wind line is highlighted by a boldface solid line.

display a broad maximum spread over the whole QBO east periods and are modulated much stronger by the SAO. There are even indications of modulations due to the SAO in the middle stratosphere. It should be noted that these fast band waves are not visible in the analyses by Tsai et al. (2004); Randel and Wu (2005) and Ratnam et al. (2006) using GPS data because their method is only able to resolve waves with periods longer than about 7-10 days.

There is a considerable contribution of the "fast" band waves already in the lower stratosphere, which can be of the same order as the contribution from the "slow" band waves during the periods when the "slow" band waves reach their maximum. During all other periods the "fast" band waves are dominant. In the upper stratosphere and above the "fast" band waves are always dominant.

For the equatorial Rossby waves the "slow" wave band shows a variation with the QBO whereas the variations of "fast" wave band variances are a mixture of QBO modulation and an annual cycle. For the Rossby-gravity waves the QBO variation is the main contribution in both wave bands, whereas the $n=0$ inertia-gravity waves show $\mathrm{QBO}$ variations in the "slow" band and no clear signatures for the "fast" band waves. Maybe this is due to the fact that the "fast" band as we have defined it is very narrow in order to exclude contributions of higher $n$ inertia-gravity waves.

Overall we can see that the equatorial wave activity in the stratosphere can be divided in two parts. One part at low equivalent depths is modulated mainly by the QBO winds, the other part at higher equivalent depths shows also annual variations or variations due to the SAO. This behavior is found in both SABER and ECMWF residual temperatures, again showing the surprising agreement between both data sets - even though ECMWF data are partly based on assimilated TOVS/ATOVS satellite measurements, which provide information at least about atmospheric waves with longer vertical wavelengths (see Sect. 2.3).

\subsection{Contribution of gravity waves}

We also calculate the temperature variances due to gravity waves by estimating the spectral background separately for each 31-day window and altitude for both the symmetric and antisymmetric spectra (see also Sect. 2.2). To avoid contamination of these background values by the equatorial wave 

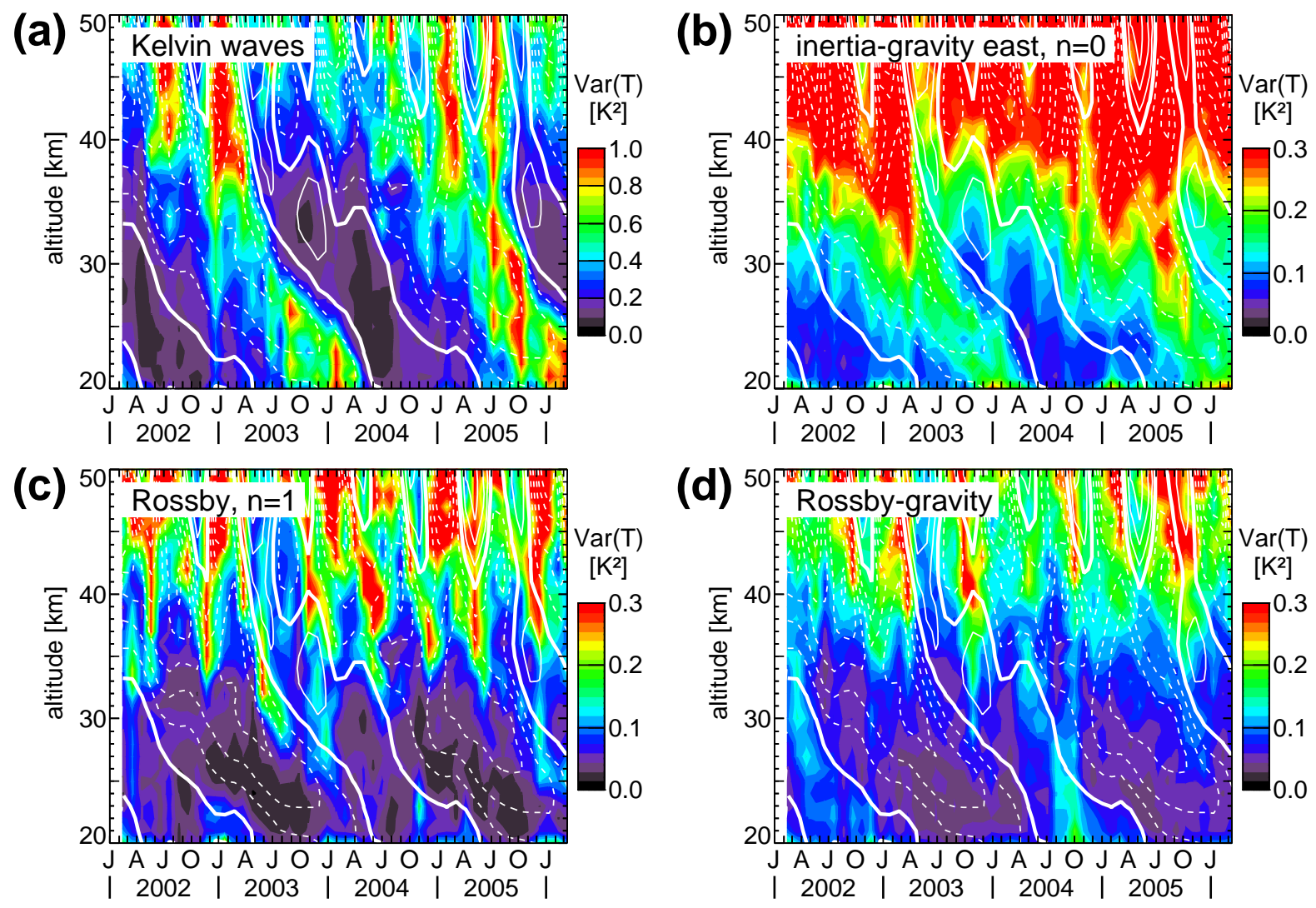

Fig. 7. Altitude-time cross-sections of SABER temperature variances integrated over the "slow" wave bands between 8 and $90 \mathrm{~m}$ equivalent depth for (a) Kelvin waves, (b) inertia-gravity waves $(n=0)$, (c) equatorial Rossby waves $(n=1)$, and (d) Rossby-gravity waves. Overplotted contour lines are zonal mean zonal wind from ECMWF averaged over the latitudes $15 \mathrm{~S}-15 \mathrm{~N}$. Contour interval is $10 \mathrm{~m} / \mathrm{s}$, solid lines indicate eastward, dashed lines westward wind. The zero wind line is highlighted by a boldface solid line.

modes we omit the tidal peaks, as well as zonal wavenumbers lower than 3 (where the main contributions of equatorial waves are located) in each of the spectra and calculate the median of the squared spectral amplitudes from the remaining spectrum.

A median is found by arranging the values in order and then selecting the one in the middle. We use this method instead of the conventional mean-value average because even in the remaining parts of the spectra there will be "outliers" (localized spectral signatures of equatorial wave modes with amplitudes much higher than the spectral background) that will high-bias the mean value of the spectral background. This is avoided by using the median because in the remaining parts of the spectra the majority of spectral values is dominated by the spectral background (see Figs. 2 and 3).

These symmetric and antisymmetric median background values are added to obtain the background value for the complete spectrum. Then this constant value is integrated over the full spectrum (multiplied by the area of the whole spectral domain resolved) and divided by two (because the values we use are squared spectral amplitudes, i.e. two times power spectral densities) to obtain the temperature variance due to gravity waves (see also Sect. 2.2).
The resulting altitude-time distribution is shown in Fig. 11 for SABER (Fig. 11a) and ECMWF (Fig. 11b).

Over the whole altitude range the variances due to gravity waves in the SABER data obviously are considerably higher than in ECMWF (please note that the color scales in Fig. 11a and $b$ are different). SABER gravity wave variances range from about $1.5-2 \mathrm{~K}^{2}$ in the lower stratosphere to about $15-$ $20 \mathrm{~K}^{2}$ in the upper stratosphere, monotonically increasing.

The range of ECMWF gravity wave variances is from about $0.5-0.7 \mathrm{~K}^{2}$ in the lower stratosphere to about $3-5 \mathrm{~K}^{2}$ in the upper stratosphere, decreasing again above $44 \mathrm{~km}$ altitude.

Some kind of cross-check for the median technique can be made by comparing the average variances at 21 and $41 \mathrm{~km}$ altitude in Fig. 11 with the background variances that were estimated from the 4-year average spectra in Sects. 2.2 and 2.4. The values obtained there are about $2 \mathrm{~K}^{2}$ and $7 \mathrm{~K}^{2}$ for SABER and $0.5-0.7 \mathrm{~K}^{2}$ and $3-4 \mathrm{~K}^{2}$ for ECMWF at 21 and $41 \mathrm{~km}$ altitude, respectively. As we can see these values are in good agreement with the average values obtained from Fig. 11 at 21 and $41 \mathrm{~km}$ altitude.

The SABER gravity wave variances show an annual cycle in the lower stratosphere which is less pronounced in the 

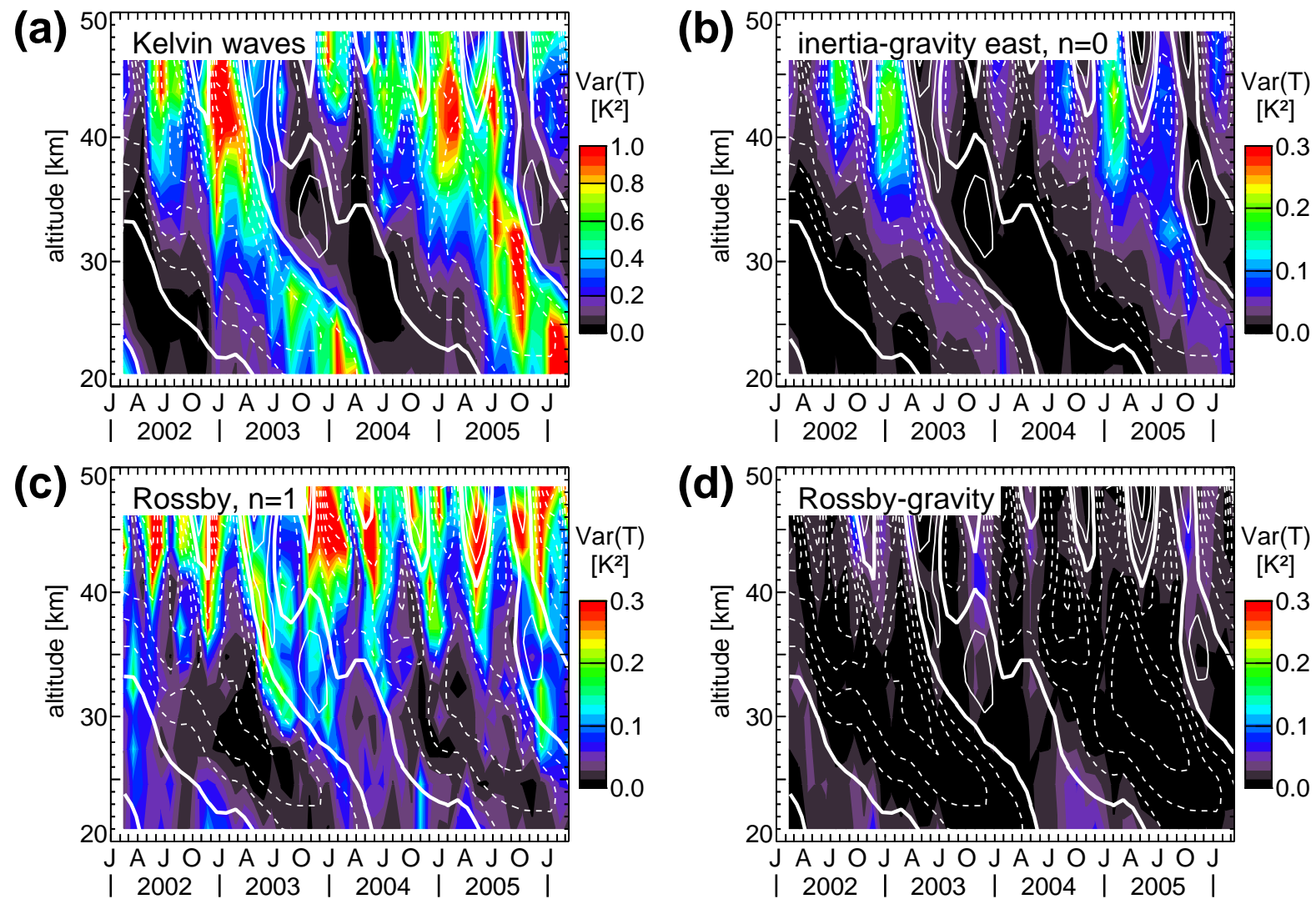

Fig. 8. Altitude-time cross-sections of ECMWF temperature variances integrated over the "slow" wave bands between 8 and $90 \mathrm{~m}$ equivalent depth for (a) Kelvin waves, (b) inertia-gravity waves $(n=0)$, (c) equatorial Rossby waves $(n=1)$, and (d) Rossby-gravity waves. Overplotted contour lines are zonal mean zonal wind from ECMWF averaged over the latitudes $15 \mathrm{~S}-15 \mathrm{~N}$. Contour interval is $10 \mathrm{~m} / \mathrm{s}$, solid lines indicate eastward, dashed lines westward wind. The zero wind line is highlighted by a boldface solid line.

equatorial waves (compare Figs. 5-10). In addition there are also variations due to the $\mathrm{QBO}$ with maximum variances at about the same times as the "slow" wave band Kelvin waves. In the upper stratosphere the gravity waves show variations due to the SAO. ECMWF variances show similar variations, but less pronounced.

This behavior agrees with previous findings from SABER residual temperatures using a different method (Krebsbach and Preusse, 2007) and from GPS radio occultation measurements (Wu, 2006; de la Torre et al., 2006). For a more detailed discussion see Sect. 4.2

Since the variation of gravity wave variances with the QBO is similar to the observed variation of Kelvin wave variances (see Figs. 5-10) the question arises whether the variation of gravity wave variances is just some contamination of the background variances by Kelvin waves. There are several reasons why the observed QBO-related variations of gravity wave variances should be real and not only an artifact.

First, from theoretical considerations a variation of gravity wave variances with the QBO would be expected. Combining Eqs. (1) and (3) in Preusse et al. (2006), we obtain the temperature amplitude limit $\hat{T}_{\text {sat }}$ critical for wave breaking:

$\hat{T}_{\mathrm{sat}}=\frac{\bar{T}}{g} N|c-\bar{u}|$

with $\bar{T}$ and $\bar{u}$ the background temperature and the background wind and $c=\omega / k$ the ground based phase speed of the wave. This equation is valid for gravity waves in mid-frequency approximation. From Eq. (10) we can see that the saturated amplitudes of gravity waves with ground based phase speeds comparable to the wind amplitude of the QBO will be modulated by the QBO winds (i.e., even gravity waves with ground based phase speeds as high as about $40 \mathrm{~m} / \mathrm{s}$ ). From Eq. (10) we can also see that in a stronger background wind gravity waves propagating against the background wind can attain larger amplitudes before the amplitude limit critical for wave breaking is reached. In QBO easterly phases the background wind is stronger than in the westerly phases. Therefore some kind of QBO modulation of gravity wave variances would be expected. In particular, an increase of temperature variances in a layer below the lines of zero zonal wind about $2 \mathrm{~km}$ thick would be expected from 

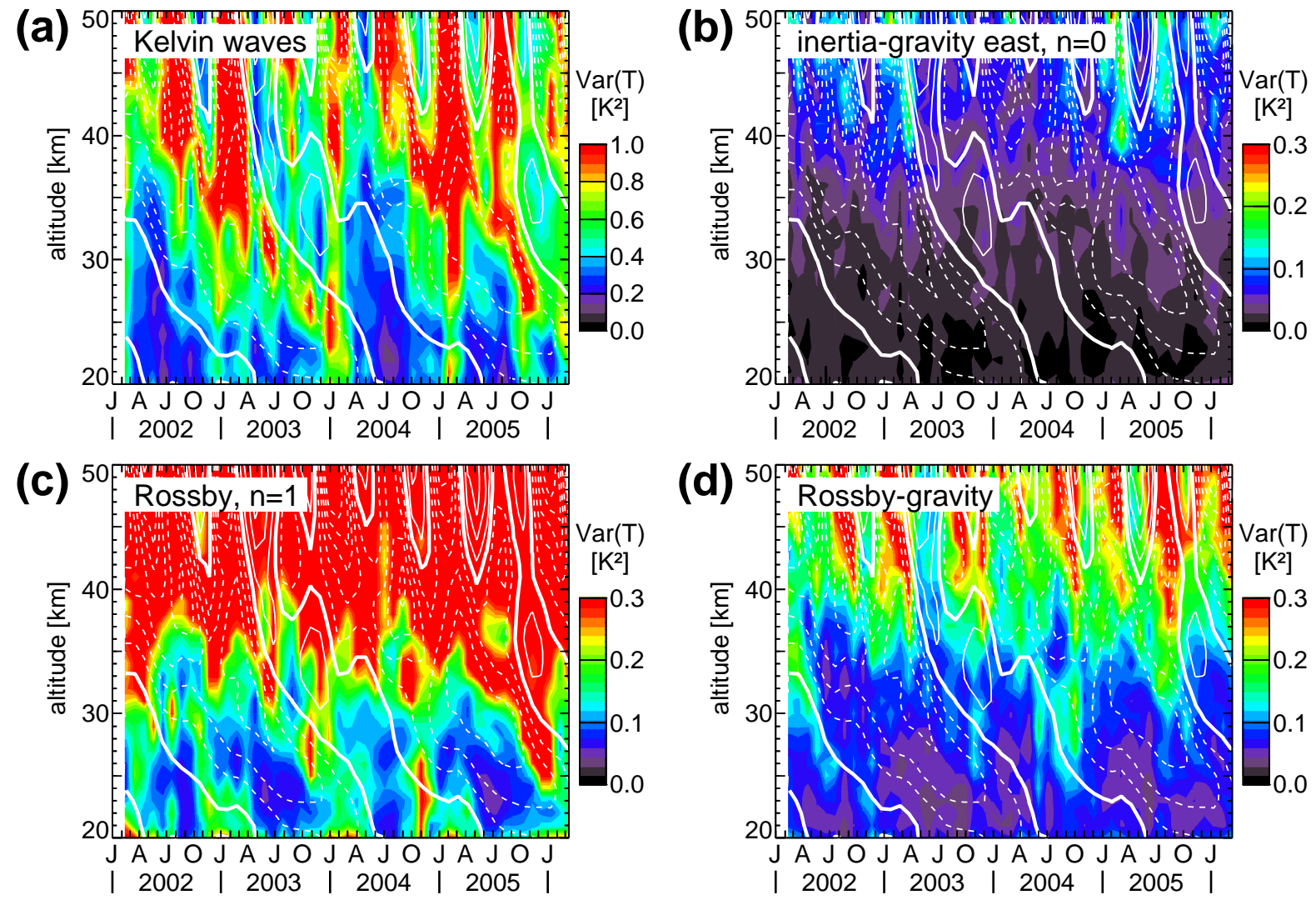

Fig. 9. Altitude-time cross-sections of SABER temperature variances integrated over the "fast" wave bands between 90 and $2000 \mathrm{~m}$ equivalent depth for (a) Kelvin waves, (b) inertia-gravity waves $(n=0)$, (c) equatorial Rossby waves ( $n=1)$, and (d) Rossby-gravity waves. Overplotted contour lines are zonal mean zonal wind from ECMWF averaged over the latitudes $15 \mathrm{~S}-15 \mathrm{~N}$. Contour interval is $10 \mathrm{~m} / \mathrm{s}$, solid lines indicate eastward, dashed lines westward wind. The zero wind line is highlighted by a boldface solid line.

amplitude growth arguments (see Randel and Wu, 2005, Appendix A). Such layers can also be found during the phases of eastward shear in the westward wind of the QBO east phases at altitudes of $20-30 \mathrm{~km}$ in the SABER gravity wave variances shown in Fig. 11a. In the ECMWF data (Fig. 11b) this is only weakly indicated.

Indeed, short period waves (gravity waves) have been found to be modulated by the QBO before in analyses of radiosonde data (Maruyama, 1994; Sato et al., 1994; Sato and Dunkerton, 1997; Vincent and Alexander, 2000). In addition, Krebsbach and Preusse (2007) found a variation of gravity wave temperature variances with the QBO using SABER satellite data but an approach different from the one used here.

Nevertheless, there might be some contamination due to Kelvin waves left in the total spectral background variances shown in Fig. 11 and we will do some kind of cross-check in the following. To further suppress the effect of Kelvin waves we estimated background variances only from frequencies $<0 \mathrm{cpd}$ in the antisymmetric spectra (again using the median method described above). By using only frequencies $<0$ cpd we also avoid contamination due to eastward propa- gating wave modes other than Kelvin waves. Since this part of the spectrum is dominated by westward propagating equatorial waves enhanced variances during the QBO westerly phases (different from the Kelvin waves) would be expected if the spectral background was contaminated by those waves.

Different from Fig. 11 where both symmetric and antisymmetric background values as well as almost the whole frequency range were used we now estimate total variances by taking twice the variances determined from only the frequency $<0 \mathrm{cpd}$ parts of the antisymmetric spectra. By doing so we assume that symmetric and antisymmetric backgrounds as well as the background for frequencies $<0 \mathrm{cpd}$ and frequencies $>0 \mathrm{cpd}$ should be all about equal (which is approximately the case) and the background variance of the original data can be estimated from the frequency $<0 \mathrm{cpd}$ part of the antisymmetric spectra alone.

These total background variances are shown in Fig. 12a for SABER and Fig. 12b for ECMWF. Especially in the SABER background variances (Fig. 12a) we still see the QBO variation similar to the variation of the Kelvin waves with the QBO. For the ECMWF variances (Fig. 12b) the QBO-related variation is also still present but even less pronounced than 

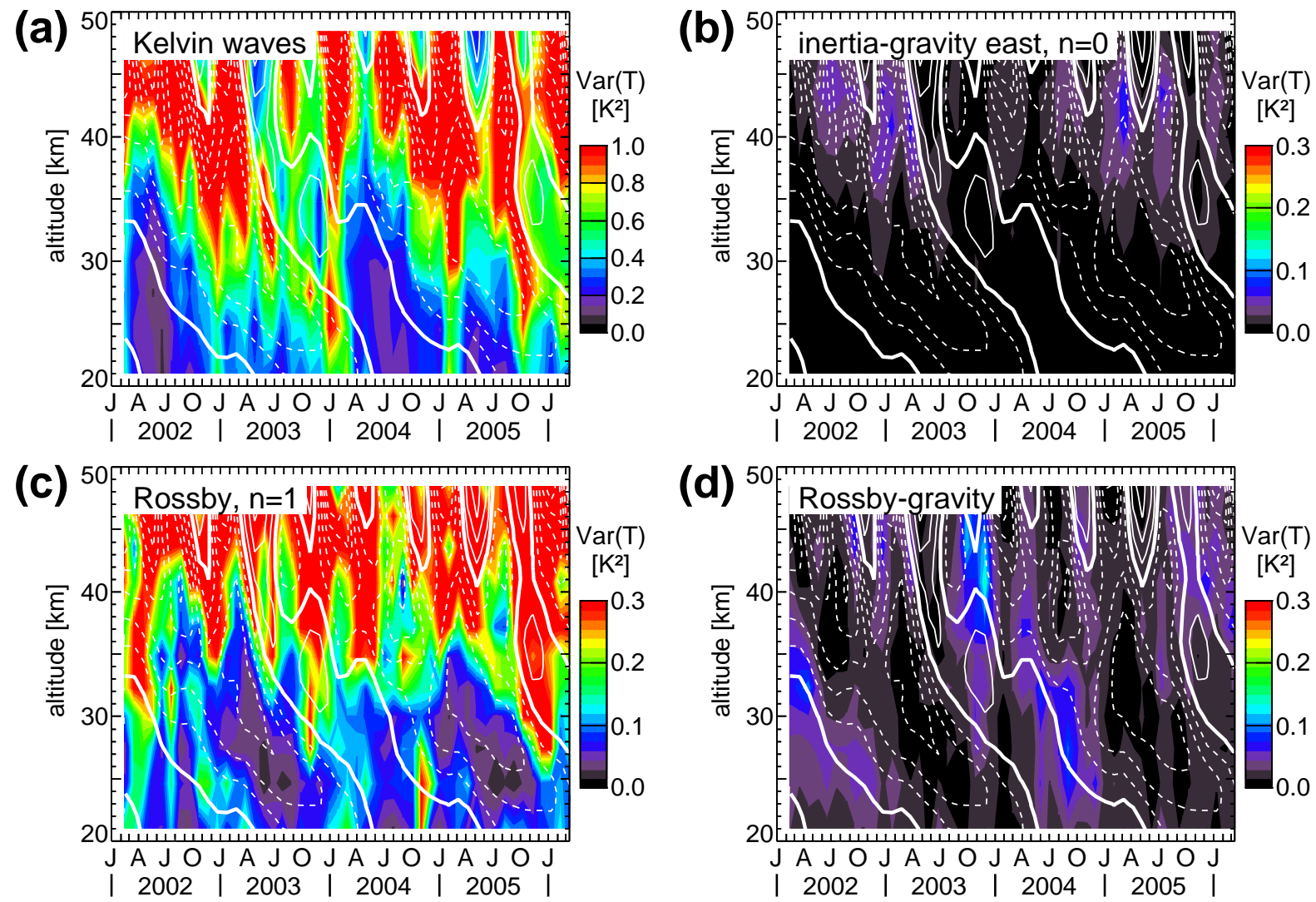

Fig. 10. Altitude-time cross-sections of ECMWF temperature variances integrated over the "fast" wave bands between 90 and $2000 \mathrm{~m}$ equivalent depth for (a) Kelvin waves, (b) inertia-gravity waves $(n=0)$, (c) equatorial Rossby waves $(n=1)$, and (d) Rossby-gravity waves. Overplotted contour lines are zonal mean zonal wind from ECMWF averaged over the latitudes $15 \mathrm{~S}-15 \mathrm{~N}$. Contour interval is $10 \mathrm{~m} / \mathrm{s}$, solid lines indicate eastward, dashed lines westward wind. The zero wind line is highlighted by a boldface solid line.
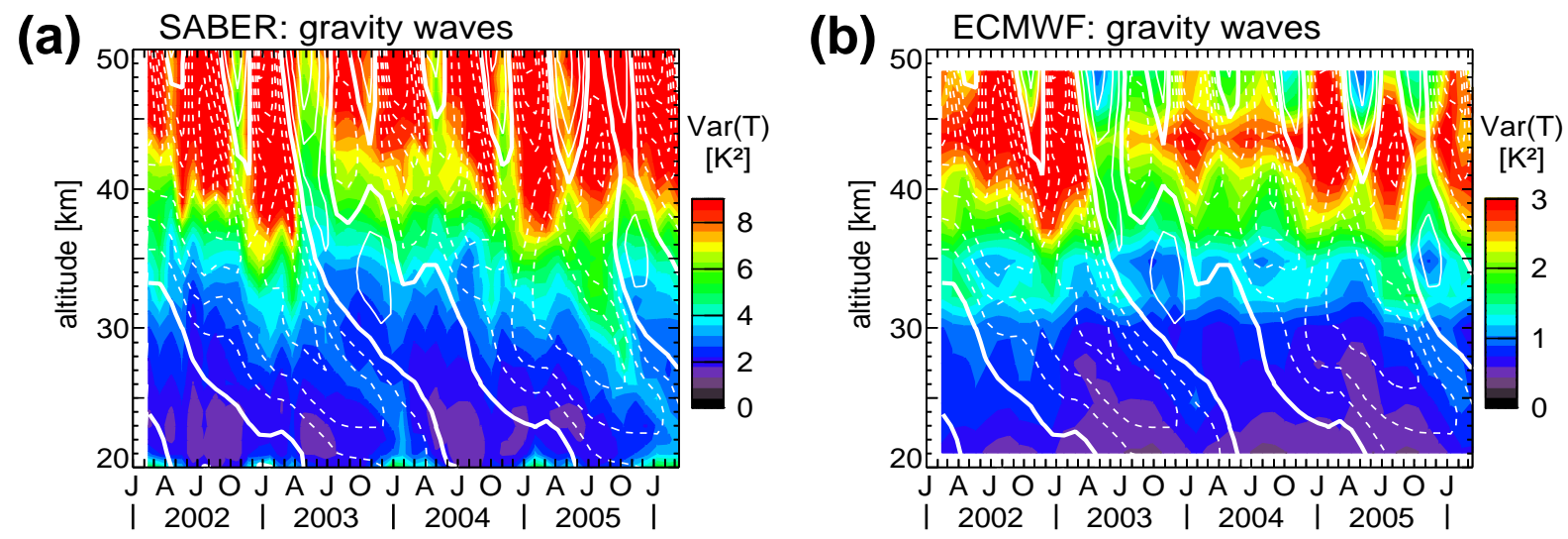

Fig. 11. Altitude-time cross-sections of SABER (a) and ECMWF (b) temperature variances due to gravity waves. The variances were determined from the spectral background in the space-time spectra. Please note that the color scales in (a) and (b) differ by a factor of 3 . Overplotted contour lines are zonal mean zonal wind from ECMWF averaged over the latitudes $15 \mathrm{~S}-15 \mathrm{~N}$. Contour interval is $10 \mathrm{~m} / \mathrm{s}$, solid lines indicate eastward, dashed lines westward wind. The zero wind line is highlighted by a boldface solid line. 
in Fig. 11b. Please note that the color scales in Fig. 12 are somewhat lower than in Fig. 11, accounting for minor asymmetries in the spectral background values.

It should be noted that for SABER we cannot rule out completely that even antisymmetric spectra and frequencies $<0 \mathrm{cpd}$ might be contaminated by aliasing effects from Kelvin waves with zonal wavenumbers $>7$ not properly resolved by the satellite sampling. However, this is not a likely effect since the spectra shown in Figs. 2 and 3 indicate that the contributions due to Kelvin waves strongly decrease at zonal wavenumbers $>5$ for $21 \mathrm{~km}$ altitude (Fig. 2) and even more for $41 \mathrm{~km}$ altitude (Fig. 3).

This information together with the fact that QBO-related variations are also found in the variances shown in Fig. 12 indicates that the QBO-related background variations found (especially for SABER) are a robust feature that can be attributed to QBO-related variations of gravity wave variances.

\section{Comparison to previous analyses}

\subsection{Equatorial waves}

As mentioned in Sects. 2.2-2.4 the general behavior of the spectral signatures observed in SABER and ECMWF temperatures in the stratosphere is as follows: In the lowermost stratosphere the spectral features are already somewhat shifted towards higher phase speeds or equivalent depths compared to the observations in the troposphere by, for example, Wheeler and Kiladis (1999) or Cho et al. (2004), which are dominated by convectively coupled equatorial waves. With increasing altitude higher phase speeds and equivalent depths (dominated by free equatorial wave modes) become even more important because slow phase speed waves will more and more encounter critical level filtering and wave dissipation. Higher phase speed waves have been observed before by, e.g., Salby et al. (1984); Hitchman and Leovy (1988); Lieberman and Riggin (1997); Garcia et al. (2005).

For a quantitative comparison, we compare our results to the analyses of GPS temperature data mentioned above (Tsai et al., 2004; Randel and Wu, 2005; Ratnam et al., 2006). In these analyses Kelvin wave activity of zonal wavenumbers 1 and 2 with periods longer than about 7-10 days is covered (Ratnam et al., 2006). These are the main contributions also present in the "slow" wave bands of SABER and ECMWF data. Therefore temperature variances of the GPS analyses can directly be compared to our "slow" Kelvin wave band variances.

The structures in the altitude/time cross-sections (Figs. 7 and 8) are quite similar to the ones shown in Ratnam et al. (2006). Peak values of temperature amplitudes in the middle stratosphere observed by Ratnam et al. (2006) are about 1$1.5 \mathrm{~K}^{2}$ as an average over the dominant wavenumber 1 and 2 components. This would result in temperature variances of about $1-2 \mathrm{~K}^{2}$ if we assume that both wavenumber 1 and 2 have the same amplitudes. This is in good agreement with the peak values of about $1-1.5 \mathrm{~K}^{2}$ for the "slow" Kelvin wave band in SABER and ECMWF data (see Figs. 7 and 8).

These values are also in good agreement with findings from radiosonde observations. For example Angell et al. (1973) find peak variances of about $2 \mathrm{~K}^{2}$ at the equator in their 12-year data set at 50 mbar for wave periods between 10 and 20 days. In another example by Sato et al. (1994) for wave periods between 8 and 20 days peak temperature variances between about 1 and $2.5 \mathrm{~K}^{2}$ in the altitude region 20 $30 \mathrm{~km}$ are found for a 15 -year data set of routine rawinsondes at Singapore. These values are somewhat higher than the $0.5-1.5 \mathrm{~K}^{2}$ in our "slow" Kelvin wave band. This difference can easily be explained because the values of our analysis represent an average over the latitudes from about $15 \mathrm{~S}-15 \mathrm{~N}$ and equatorial wave activity decreases towards higher latitudes. In addition, our "slow" Kelvin wave band not fully coincides with the waves resolved by the radiosonde analyses.

\subsection{Gravity waves}

The variances of gravity waves shown in Fig. 11a are derived from space-time spectra of SABER residual temperatures. The values obtained can be compared to values derived by Preusse et al. (2006) using a different method based on a vertical harmonic analysis using altitude profiles of SABER residual temperatures. These residual temperatures were obtained by subtracting a background distribution estimated with a zonal wavenumber 0-6 Kalman filter.

For the equatorial region the values given in Fig. 2 of Preusse et al. (2006) for August 2003 are about $7 \mathrm{~dB}$ of temperature squared amplitudes at about $20 \mathrm{~km}$ altitude and about $14 \mathrm{~dB}$ at about $50 \mathrm{~km}$ altitude, corresponding to squared amplitudes of about $5 \mathrm{~K}^{2}$ and $25 \mathrm{~K}^{2}$, respectively, i.e., temperature variances of about $2.5 \mathrm{~K}^{2}$ and $12.5 \mathrm{~K}^{2}$, respectively.

The values we obtain from the space-time spectra for $\mathrm{Au}$ gust 2003 are about $2 \mathrm{~K}^{2}$ at $20 \mathrm{~km}$ altitude and about $10 \mathrm{~K}^{2}$ for $50 \mathrm{~km}$ altitude (see Fig. 11a). This is a very good agreement, taking into account that the two methods for determining gravity wave temperature variances are very different.

Indications that short-period (small-scale) waves show modulations related to the QBO were found before in radiosonde data by, for example, Maruyama (1994); Sato et al. (1994); Sato and Dunkerton (1997); Vincent and Alexander (2000). Sato and Dunkerton (1997) estimated momentum fluxes for both Kelvin waves and 1-3 day period gravity waves based on an 8-year time series of radiosonde observations at Singapore and the association between the wave fluxes and the QBO was investigated. Vincent and Alexander (2000) carried out a similar study for small-scale waves based on a 6-year data set of radiosonde observations at Cocos Islands. In addition to strong annual and interannual 

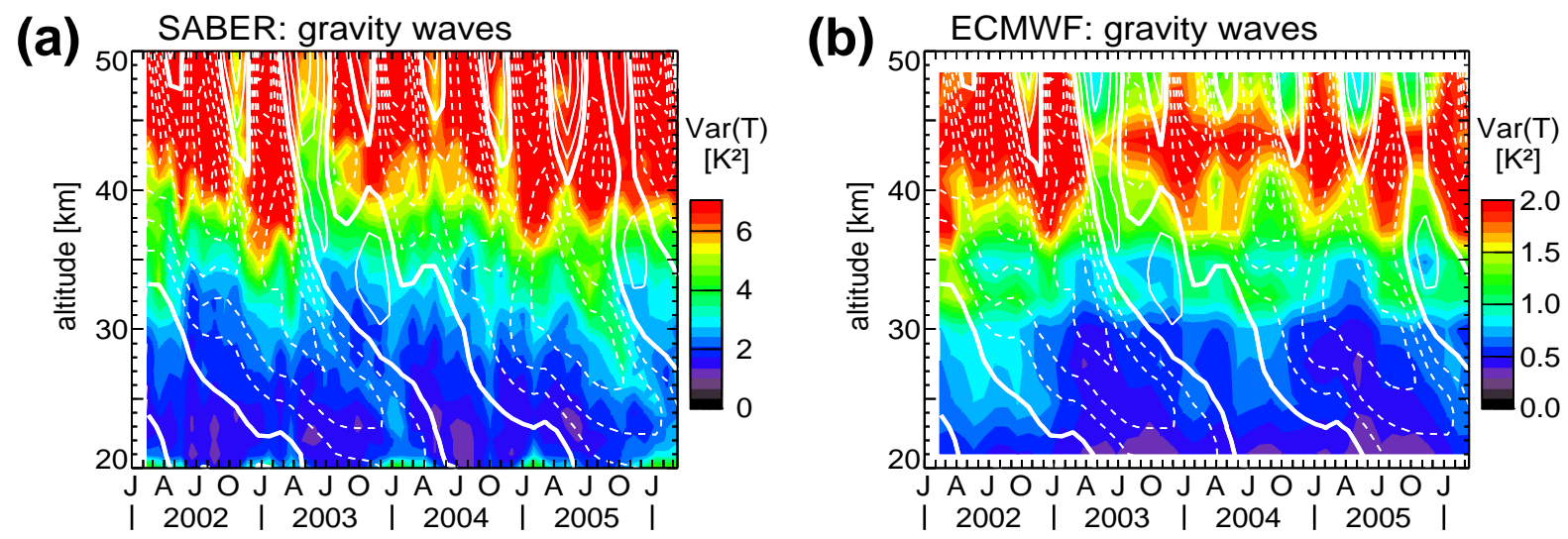

Fig. 12. Altitude-time cross-sections of SABER (a) and ECMWF (b) temperature variances due to gravity waves. Different from Fig. 11 the variances were estimated from the spectral background in the antisymmetric space-time spectra from frequencies $<0$ only. Please note that the color scales in (a) and (b) differ by a factor of about 3 and are also somewhat different from Fig. 11. Overplotted contour lines are zonal mean zonal wind from ECMWF averaged over the latitudes $15 \mathrm{~S}-15 \mathrm{~N}$. Contour interval is $10 \mathrm{~m} / \mathrm{s}$, solid lines indicate eastward, dashed lines westward wind. The zero wind line is highlighted by a boldface solid line.

variations they also find variations related to the QBO in wave energy and zonal momentum fluxes.

Also investigations of QBO-related variations based on satellite data have been carried out before. The temporal evolution of variances due to wave activity in the equatorial region including gravity waves has been investigated before by, for example, Wu (2006) and de la Torre et al. (2006) using GPS radio occultation measurements. The observed pattern is very similar to the one observed for Kelvin waves. In these analyses the horizontal structure of the waves is completely neglected. Only vertical smoothing of the measured temperature altitude profiles is applied to separate residual temperatures from the background. Therefore the results show a mixture between global equatorial wave modes (mainly Kelvin waves) and gravity waves. This is improved in our results presented in Sect. 3.2. We cleanly separate the contribution due to gravity waves from global-scale equatorial wave modes by determining the spectral background in the spacetime spectra (see Sect. 3.2).

In an analysis by Krebsbach and Preusse (2007) based on SABER gravity wave squared amplitudes annual variations as well as QBO related variations were found in the lower stratosphere and variations due to the SAO were found in the upper stratosphere. This general behavior can also be seen in the results presented in Sect. 3.2, confirming the finding that also gravity waves are modulated by the QBO. The amplitude of the QBO variation found by Krebsbach and Preusse (2007) is about $0.2 \mathrm{~K}$ in the stratosphere.

In Sect. 2.2 we have found an average temperature variance of about $2 \mathrm{~K}^{2}$ due to gravity waves in the lower stratosphere (i.e., amplitudes of about $2 \mathrm{~K}$ ). An estimate for maximum and minimum amplitudes due to the QBO modulation is then given by $2 \mathrm{~K} \pm 0.2 \mathrm{~K}$ using the QBO amplitude of $0.2 \mathrm{~K}$ by Krebsbach and Preusse (2007). Therefore there should be a modulation of the temperature variances between $1.8^{2} \mathrm{~K}^{2} / 2$ and $2.2^{2} \mathrm{~K}^{2} / 2$ due to the QBO, i.e. between about $1.6 \mathrm{~K}^{2}$ and $2.4 \mathrm{~K}^{2}$ which is in good agreement with the values of about 1.8 and $3 \mathrm{~K}^{2}$ which can be seen from Fig. 11a.

The amplitude of the annual cycle seen by Krebsbach and Preusse (2007) is between about 0.05 and about $0.2 \mathrm{~K}$ in the equatorial region between $15 \mathrm{~S}$ and $15 \mathrm{~N}$. The distribution is not centered at the equator. An average amplitude that could be compared with our results would be about $0.1 \mathrm{~K}$ around $25 \mathrm{~km}$ altitude. Repeating the above estimation results in minimum and maximum variances of about 1.8 and $2.2 \mathrm{~K}^{2}$ that would be expected according to Krebsbach and Preusse (2007). This means the annual variation should be less pronounced than the variation due to the QBO, and this is also what we find from Fig. 11a.

It should be noted that the above estimates for the QBO and annual variations of gravity wave variances are not more than some kind of cross-check. In addition, the stronger annual cycle observed at the lowermost altitudes in SABER data is not seen in GPS measurements (e.g., de la Torre et al., 2006) and could be an indication that at the lowermost altitudes the SABER temperatures might be influenced by clouds.

\section{Examples for equatorial wave activity during QBO easterly and $\mathrm{QBO}$ westerly phases}

For field campaigns meteorological analyses like, for instance, the ECMWF analyses are of great importance for mission planning during measurement campaigns as well as for the analysis of the collected data. Therefore it is an important information how reliable, for example, the horizontal 
and temporal distribution of equatorial waves is represented in the meteorological data.

One method often used to get an overview of the spatial and temporal variation are Hovmoeller plots, i.e., 2-D plots of a meteorological parameter versus longitude and time.

\subsection{QBO easterly phase (period of the SCOUT-O3 tropical aircraft campaign)}

First, we present the example of equatorial wave activity during the SCOUT-O3 tropical aircraft campaign in Darwin/Australia during November and December 2005. During this period we are in QBO easterly phase at $21 \mathrm{~km}$ altitude.

For the period of this measurement campaign the question of the reliability of meteorological analyses like ECMWF is of particular interest: A pronounced Kelvin wave is found in the ECMWF data and it is being discussed whether this Kelvin wave has a large impact on the dehydration of the tropical tropopause region during the period of the measurement campaign (Brunner et al., 2007).

Again, we average over the latitudes $15 \mathrm{~S}-15 \mathrm{~N}$. But different from Sects. 2-4 we use 91-day time windows for the space-time spectral analysis instead of 31-day windows to avoid small inconsistencies at the transitions between the 31-day windows. The results are very similar. For a better comparison between SABER and ECMWF results we therefore chose to calculate the Hovmoeller diagram from only one single (longer) time window, containing the whole time period shown. To get more reliable results also for the weaker equatorial wave modes like equatorial Rossby waves or Rossby-gravity waves we taper the data to zero at both ends of the time windows (over 10 days at each end) using a split cosine-bell window.

Tapering was not possible for the results shown in Sects. 2-4 because tapering reduces the variances with respect to the original time series. But to allow a more accurate comparison of the wave phases on a limited part of the time window tapering can reduce the spectral leakage due to edge effects caused by the finite length of the time windows.

Figure 13a shows a Hovmoeller plot of undetrended ECMWF temperatures at $21 \mathrm{~km}$ altitude, averaged over the latitudes $15 \mathrm{~S}-15 \mathrm{~N}$ in the period from 27 October until 26 December, 2005. By averaging the temperatures over a latitude band symmetric with respect to the equator antisymmetric equatorial waves cancel out and we can compare the resulting average temperature distribution only with symmetric equatorial wave analyses.

Figure 13d shows residual ECMWF temperatures averaged over the latitudes $15 \mathrm{~S}-15 \mathrm{~N}$ which can also be compared to the symmetric wave modes, whereas Fig. $13 \mathrm{~g}$ shows residual ECMWF temperatures averaged over $15 \mathrm{~S}-15 \mathrm{~N}$ antisymmetrically, i.e., the sign is reversed for residual temperatures south of the equator before averaging. Therefore Fig. $13 \mathrm{~g}$ can be compared with the signatures of the antisymmetric wave modes.
Apart from some smaller scale fluctuations due to the diurnal cycle and small scale processes the main feature in Fig. 13a apparently is a mixture of eastward propagating waves. The eastward propagation direction can be seen from the phase fronts which are tilted from the upper left towards the lower right. This information together with the fact that Kelvin waves are the most dominant process in tropical temperatures we can infer that these temperature structures are caused by a mixture of Kelvin waves.

This assumption can be confirmed by Fig. 13b and c. These two figures show the residual temperatures obtained by inverting the power spectra of the equatorial wave analysis in the "total" Kelvin wave band between 8 and $2000 \mathrm{~m}$ equivalent depth for zonal wavenumbers $1-6$. Figure $13 \mathrm{~b}$ and c shows the residual temperatures obtained from ECMWF and SABER, respectively.

Again, we find good agreement between the SABER and the ECMWF spectral analyses. Deviations between Fig. 13b and $\mathrm{c}$ are less than about $1.5 \mathrm{~K}$ maximum and about $0.3 \mathrm{~K}$ on average. At the same time the difference between maximum and minimum values in Fig. $13 \mathrm{~b}$ and $\mathrm{c}$ is about $6 \mathrm{~K}$ and the average deviation from zero is about $0.7 \mathrm{~K}$ for both data sets if unsigned absolute values are taken. This means that in the lower stratosphere ECMWF analyses do not only agree very well with the SABER temperature variances, they are also able to reproduce the temporal evolution of zonal temperature structures very well.

The Kelvin waves observed have pronounced periods of about 10-15 days, and indeed, there is a mixture of Kelvin waves leading to the observed residual temperatures of about $\pm 3 \mathrm{~K}$ maximum. If the temperature variances were dominated by a single monochromatic Kelvin wave the temperature residuals shown in Fig. 13 would show sinusoidal variations over the whole longitude range with constant amplitude, independent of longitude. Different from this we find no clear coherent sinusoidal structure over the whole longitude range in the residual temperatures shown. In addition, on average, residual temperatures are lower for longitudes $<0$ deg. In particular, residual temperatures are enhanced at longitudes $50 \mathrm{E}-180 \mathrm{E}$, i.e., in the Darwin region where the SCOUT-O3 measurement campaign took place. This indicates that not only one single sinusoidal wave is responsible for the temperature residuals observed. There has to be some kind of superposition of different Kelvin waves, involving different zonal wavenumbers and frequencies.

If we compare Fig. 13b and c with Fig. 13a we can see that most of the temperature variations symmetric with respect to the equator can be explained by Kelvin waves. (Please note that there is a temperature cooling trend in Fig. 13a masking some of the relative structures.) From Figs. 5-10 we can see that strong variations due to Kelvin waves could be expected because the SCOUT-O3 campaign in Darwin took place during a QBO east period with enhanced Kelvin wave activity in the lower stratosphere. 
(a) ECMWF: undetrended temp., symm.T $[\mathrm{K}]$

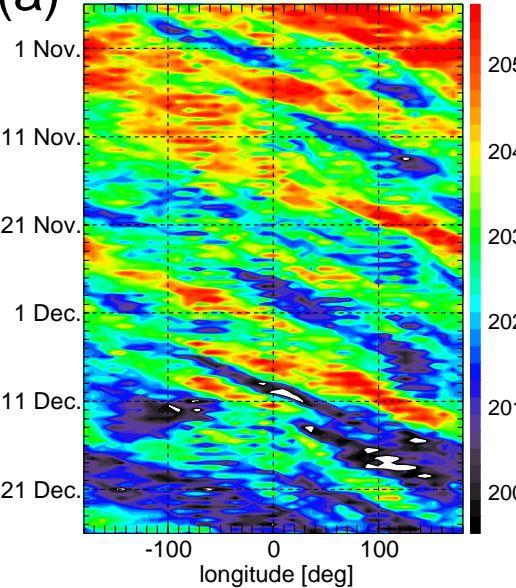

(d) ECMWF: residual temp., symm. dT [K]

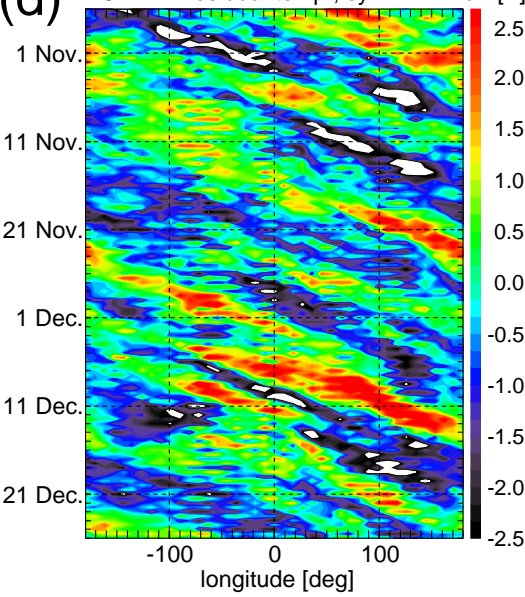

(g) ECMWF: residual temp., antisymm. dT [K]

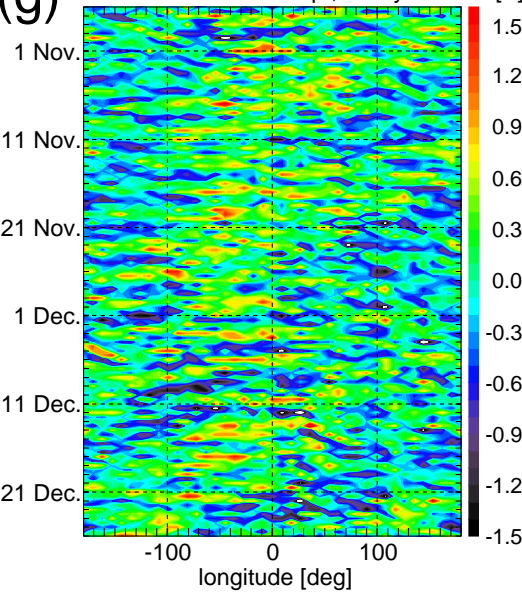

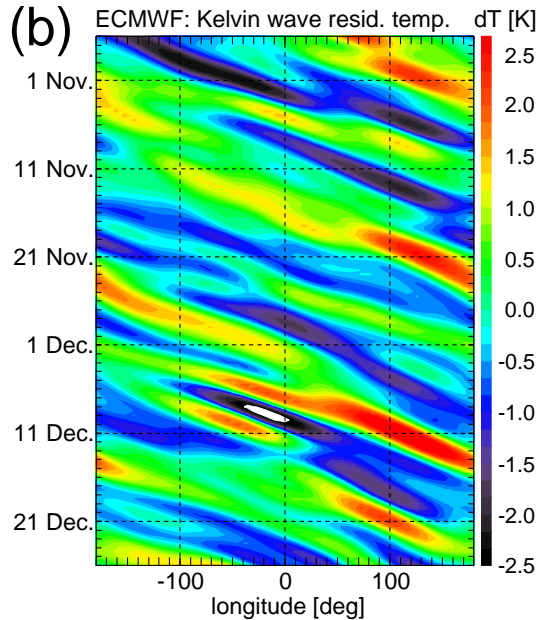

(e) ECMWF: Rossby, $n=1$ resid. temp. dT $[\mathrm{K}]$

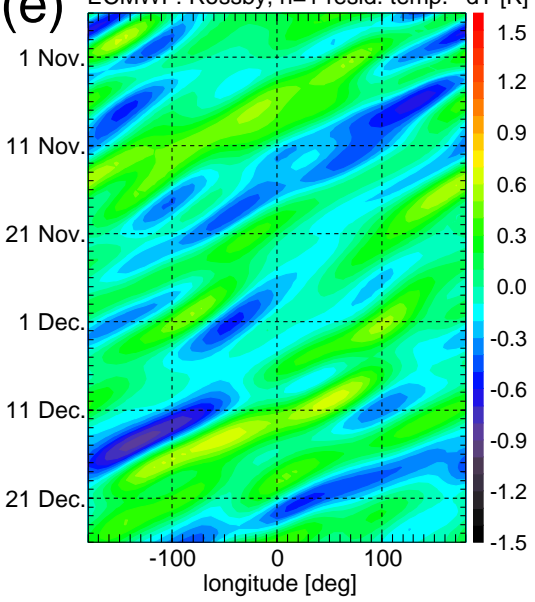

(h) ECMWF: Rossby-gravity resid. tempdT [K]

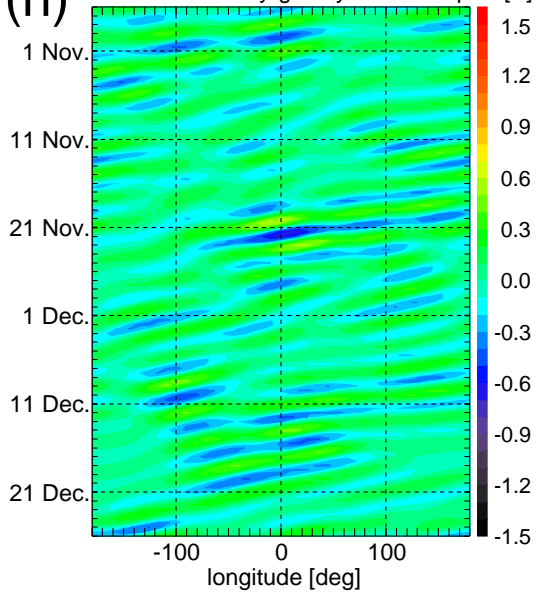

(C) SABER: Kelvin wave resid. temp. dT $[\mathrm{K}]$

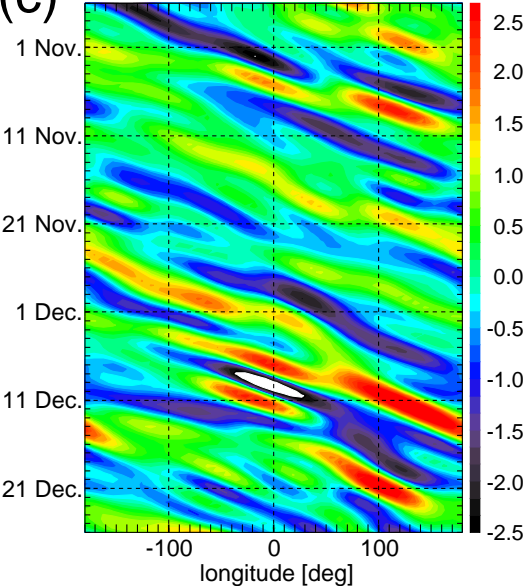

(f) SABER: Rossby, $n=1$ resid. temp. dT $[\mathrm{K}]$

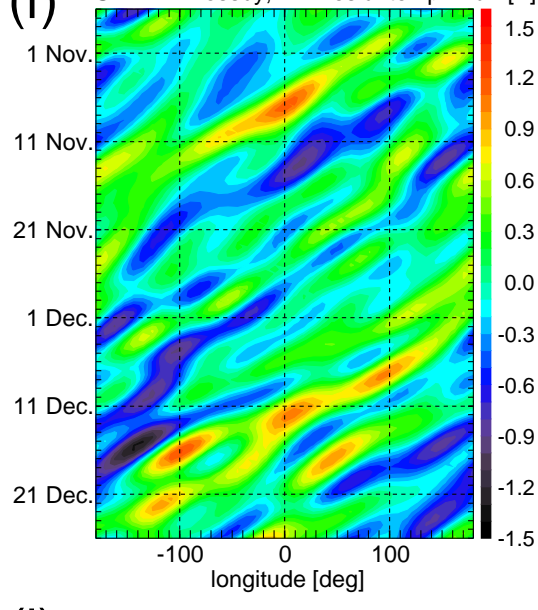

(i) SABER: Rossby-gravity resid. temp.dT [K]

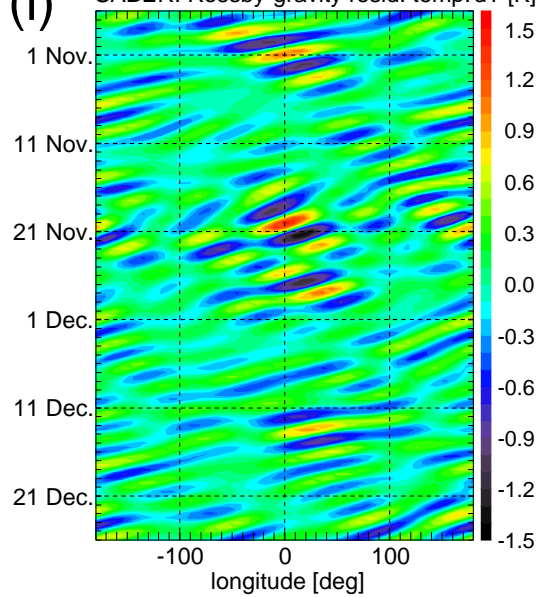

Fig. 13. Hovmoeller diagrams at $21 \mathrm{~km}$ altitude in QBO east phase for the period of the SCOUT-O3 tropical aircraft measurement campaign from 27 October until 26 December 2005. Shown are $15 \mathrm{~S}-15 \mathrm{~N}$ averages of (a) undetrended ECMWF temperatures, (d) residual ECMWF temperatures, (g) residual ECMWF temperatures averaged "antisymmetrically". Also shown are residual temperatures derived from the space-time spectral analysis for the spectral bands between 8 and $2000 \mathrm{~m}$ equivalent depth ("slow" plus "fast" wave bands) for the (b) ECMWF and (c) SABER Kelvin wave bands, (e) ECMWF and (f) SABER Rossby $n=1$ wave bands, and (h) ECMWF and (i) SABER Rossby-gravity wave bands. 
Figure $13 \mathrm{e}$ and $\mathrm{f}$ shows the signatures of equatorial Rossby waves (the $n=1$ symmetric wave mode) and Fig. 13h and $i$ the signatures of Rossby-gravity waves for ECMWF and SABER, respectively. Please note that the color scales are different from Fig. 13b and c because Rossby and Rossbygravity waves have lower amplitudes.

In the residual temperatures (Fig. 13d and g) we do not find obvious signatures of equatorial Rossby waves and Rossbygravity waves. Since the variances for those wave modes in Figs. 5 and 6 are low in November and December 2005 this is as expected. Consequently, the amplitudes found in Fig. 13e, $\mathrm{f}, \mathrm{h}$, and $\mathrm{i}$ are relatively small compared to the Kelvin wave amplitudes.

There is a certain agreement between the ECMWF and the SABER distributions of equatorial Rossby and Rossbygravity waves. But there are also significant differences. In particular, the SABER residual temperatures found in the Rossby $(n=1)$ and Rossby-gravity wave bands are considerably higher and somewhat noisier than in ECMWF. This most likely can be attributed to the spectral background due to gravity waves present in the SABER spectra. This spectral noise overlays the signatures of Rossby $(n=1)$ and Rossbygravity waves and can probably explain most of the differences between SABER and ECMWF.

\subsection{QBO westerly phase}

Figure 14 shows the same as Fig. 13 but for the period from 12 October until 11 December 2004. During this period we are in QBO westerly phase at $21 \mathrm{~km}$ altitude.

From Figs. 5-10 we can see that in this period there is enhanced activity of equatorial Rossby waves $(n=1)$ as well as enhanced variances due to Rossby-gravity waves. This can also be seen in Fig. 14e and $f$ where (different from Fig. 13e and f) we find a very pronounced Rossby $n=1$ wave with a period of about 25 days. Also the activity of Rossby-gravity waves is enhanced in Fig. 14h and i with respect to the QBO easterly phase (see Fig. 13h and i).

There is good agreement between the Rossby wave structures found in ECMWF and SABER. The Rossby-gravity wave signatures are qualitatively the same in Fig. 14h and $i$ but there are differences in details which can probably be attributed to distortions by the spectral background due to gravity waves which can be as high as about $50 \%$ of the Rossby-gravity wave variances (see Fig. 5).

However, the agreement between SABER and ECMWF Rossby-gravity waves and Rossby $n=1$ waves is much better than in Fig. 13h and i and Fig. 13e and f. First, the amplitudes of the Rossby-gravity waves and Rossby $n=1$ waves are higher in the period from October 12 until December 112004 , and, second, at $21 \mathrm{~km}$ altitude temperature variances due to gravity waves are much lower (about $2 \mathrm{~K}^{2}$, see Fig. 11a) than in the period from 27 October until 26 December 2005 (about $3 \mathrm{~K}^{2}$, see also Fig. 11a).
It should also be noted that even during the QBO westerly phase there is still a large signal due to Kelvin waves (Fig. 14b and c) - lower than during the QBO easterly phase (see Sect. 5.1) but still as large as the variations due to the Rossby $n=1$ wave during the QBO westerly phase.

Both the signatures of the Kelvin waves and the Rossby $n=1$ waves can be found in the symmetric residual temperatures from ECMWF (see Fig. 14a, d). Even the signatures of the Rossby-gravity waves can be found in the antisymmetric ECMWF residual temperatures (see Fig. 14g) in spite of the relatively low amplitudes on average.

For all wave modes shown at $21 \mathrm{~km}$ altitude we find qualitatively good agreement between SABER and ECMWF not only in the wave amplitudes but also in the phases of the waves for both QBO easterly and westerly phases. Some disagreements can be explained by the gravity wave background which is much higher in the SABER data than in ECMWF. Certain agreement between SABER and ECMWF was expected since TOVS/ATOVS satellite data are assimilated in the ECMWF operational analyses. Nevertheless, it is somewhat surprising that already in the lower stratosphere there is such good agreement between SABER and ECMWF, taking into account that in the lower stratosphere equatorial waves on average have lower equivalent depths and, consequently, shorter vertical wavelengths. Since TOVS/ATOVS data are measured in nadir viewing geometry with broad vertical weighting functions especially short vertical wavelengths should be somewhat degraded in the TOVS/ATOVS measurements entering the ECMWF analyses.

\section{Conclusions}

We carried out an analysis for equatorial waves based on four-year (Feb 2002 until March 2006) data sets of SABER and ECMWF temperatures. We divided the equatorial waves into symmetric and antisymmetric wave modes, similar to the analysis by Wheeler and Kiladis (1999). This method performed well for both the asynoptic data set of SABER satellite measurements as well as the ECMWF meteorological analyses, given on a regular grid. Both data sets are obviously capable to resolve longer period waves as well as short period waves, at least down to periods of about 2 days.

The spectral signatures of Kelvin waves, equatorial Rossby waves, inertia-gravity waves $(n=0)$, as well as Rossby-gravity waves can be identified clearly throughout the stratospheric altitude range of $20-50 \mathrm{~km}$ considered in this paper. We find that the equatorial wave activity in a slow phase speed wave band between 8 and $90 \mathrm{~m}$ equivalent depth is mainly modulated by the QBO. Different from this the waves at higher equivalent depths (90-2000 m) show less pronounced variation due to the QBO. Also effects of SAO and annual cycle can be found. For Kelvin waves the contribution of these "fast" waves cannot be neglected even in the lower stratosphere. 
(a)

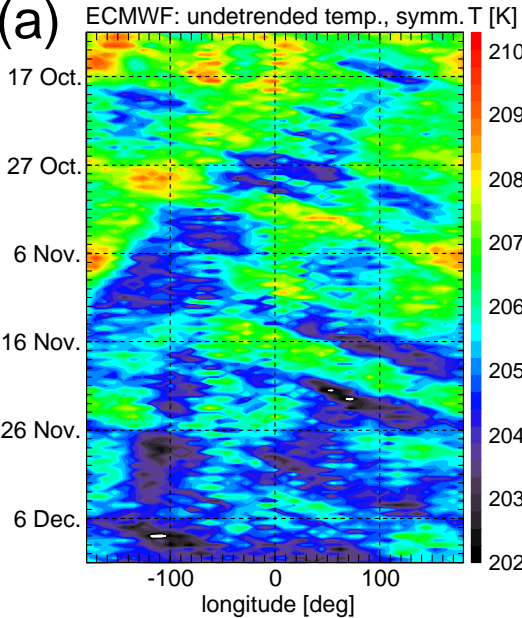

(d) ECMWF: residual temp., symm. dT [K]

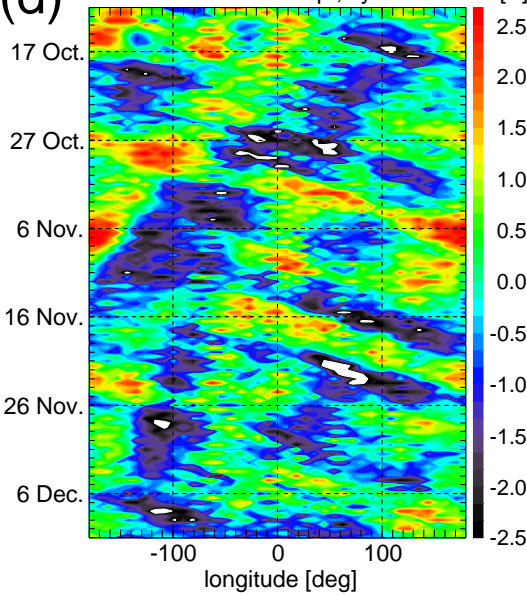

(g) ECMWF: residual temp. antisymm. dT [K]

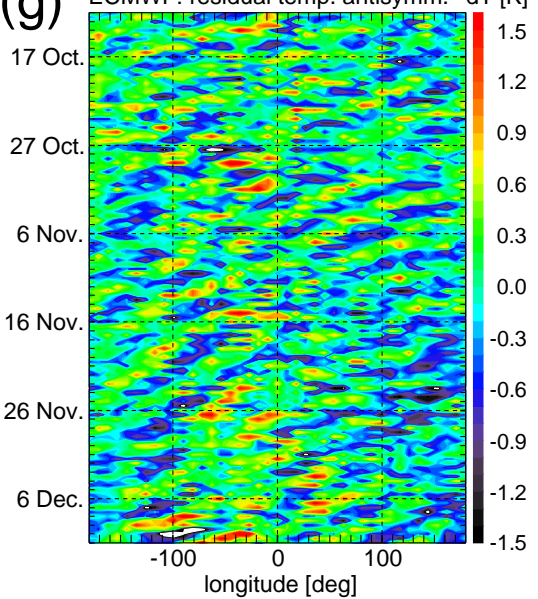

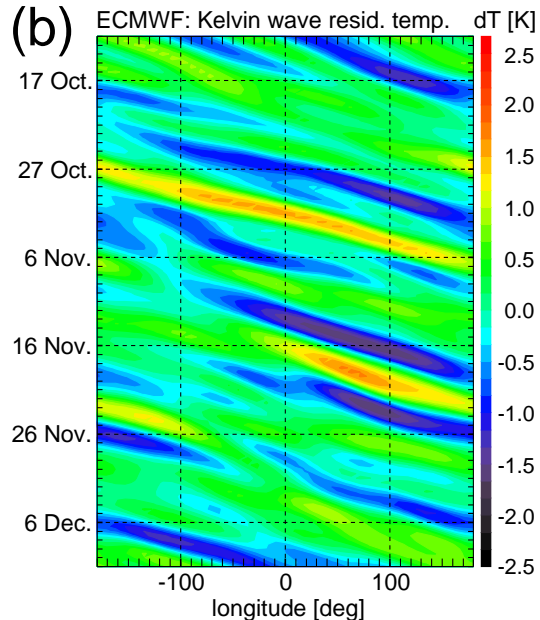

(e) ECMWF: Rossby, $\mathrm{n}=1$ resid. temp. dT [K]

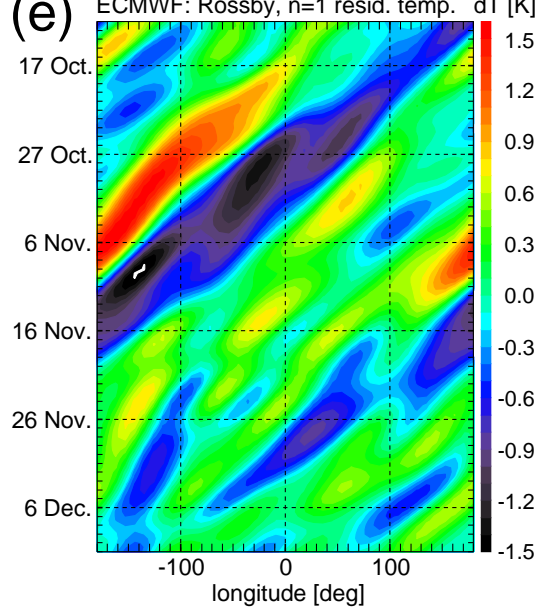

(h) ECMWF: Rossby-gravity resid. tempdT [K]

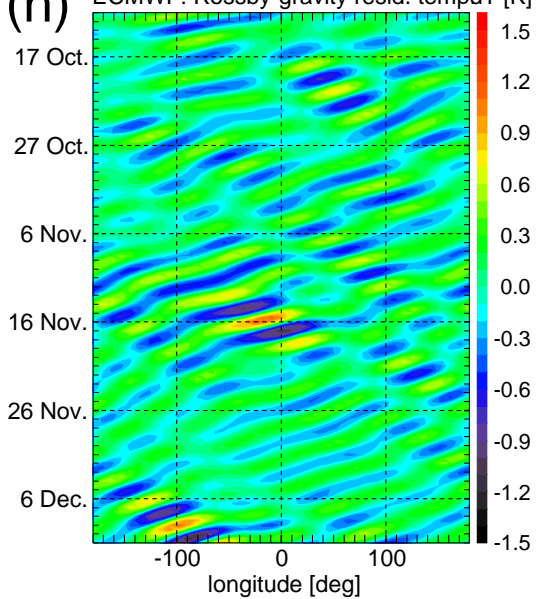

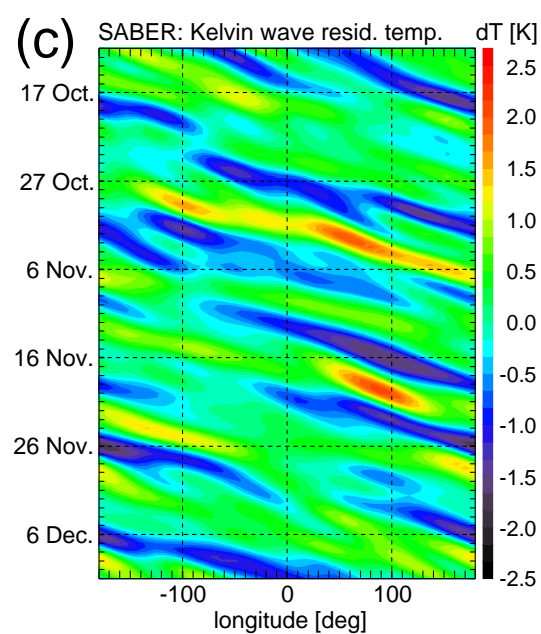

(f) SABER: Rossby, $n=1$ resid. temp. dT [K]

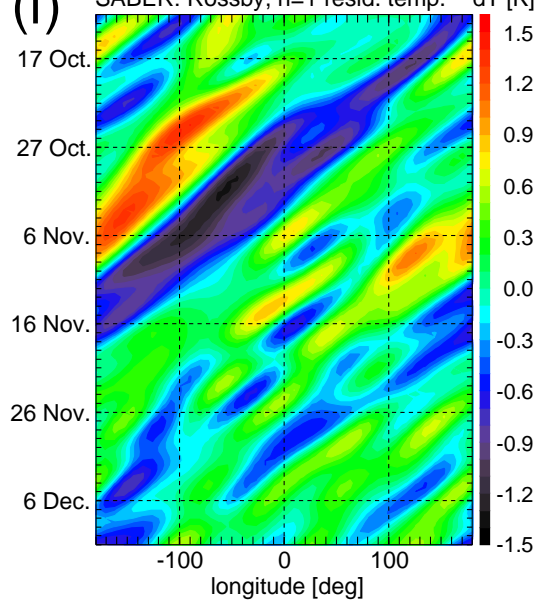

(i) SABER: Rossby-gravity resid. temp. dT [K]

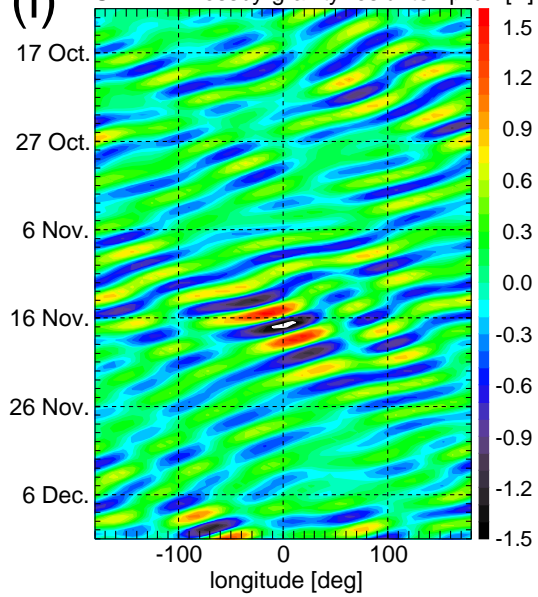

Fig. 14. Hovmoeller diagrams at $21 \mathrm{~km}$ altitude in QBO west phase for the period from 12 October until 11 December 2004 . Shown are $15 \mathrm{~S}-15 \mathrm{~N}$ averages of (a) undetrended ECMWF temperatures, (d) residual ECMWF temperatures, (g) residual ECMWF temperatures averaged "antisymmetrically". Also shown are residual temperatures derived from the space-time spectral analysis for the spectral bands between 8 and 2000 m equivalent depth ("slow" plus "fast" wave bands) for the (b) ECMWF and (c) SABER Kelvin wave bands, (e) ECMWF and (f) SABER Rossby $n=1$ wave bands, and (h) ECMWF and (i) SABER Rossby-gravity wave bands. 
We find good agreement between our analyses and previous studies based on satellite data (e.g., analysis of GPS temperatures by Ratnam et al., 2006) as well as analyses based on radiosonde measurements (e.g., Angell et al., 1973). It is also remarkable that there is very good agreement between the SABER and ECMWF analyses. Only in the upper stratosphere ECMWF tends to overestimate Kelvin wave components (in some cases by more than 50\%). There is agreement not only in the spectra, but also in the temporal evolution of the temperature variances and even in the longitudinal distribution of residual temperatures and their temporal evolution in the lower stratosphere. This has been demonstrated at $21 \mathrm{~km}$ altitude in QBO westerly phase (period OctoberDecember 2004) as well as QBO easterly phase (November/December 2005, period of the SCOUT-O3 tropical aircraft campaign in Darwin/Australia) for different equatorial wave modes (Kelvin, equatorial Rossby $(n=1)$, and Rossbygravity waves).

From our space-time analysis we are also able to derive the temperature variances of gravity waves from the spectral background for both SABER and ECMWF space-time spectra. The results for SABER are in good agreement with previous results by Preusse et al. (2006) and Krebsbach and Preusse (2007) while ECMWF underestimates the variances due to gravity waves by a factor of about 3 .

This shows on one hand that the SABER data are an excellent data set providing high-resolution data with large spatial coverage and on the other hand that although ECMWF tends to overestimate Kelvin wave components at higher altitudes and small scale fluctuations are underrepresented the ECMWF data are also able to reliably reproduce measurements of equatorial waves at least in the lower stratosphere, making ECMWF a valuable tool accompanying measurement campaigns.

Acknowledgements. The current work was supported by the European Union's 6th framework program within the SCOUT-O3 (GOCE-CT-2004-505390) project. Thanks also goes to the European Centre for Medium-Range Weather Forecasts (ECMWF) for providing the global ECMWF analyses used and to N. Bormann from ECMWF for helpful information.

Edited by: P. Haynes

\section{References}

Alexander, M. J., Holton, J. R., and Durran, D. R.: The gravity wave response above deep convection in a squall line simulation, J. Atmos. Sci., 52, 2212-2226, 1995.

Angell, J. K., Cotton, G. F., and Korshover, J.: A climatological analysis of oscillations of Kelvin wave period at $50 \mathrm{mb}$, J. Atmos. Sci., 30, 13-24, 1973.

Baldwin, M. P., Gray, L. J., Dunkerton, T. J., Hamilton, K., Haynes, P. H., Randel, W. J., Holton, J. R., Alexander, M. J., Hirota, I., Horinouchi, T., Jones, D. B. A., Kinnersley, J. S., Marquardt, C.,
Sato, K., and Takahashi, M.: The quasi-biennial oscillation, Rev. Geophys., 39, 179-229, 2001.

Bergman, J. W. and Salby, M. L.: Equatorial wave activity derived from fluctuations in observed convection, J. Atmos. Sci., 51, 3791-3806, 1994.

Brunner, D., Peter, T., Schiller, C., Krebsbach, M., Sitnikov, N. M., and Mezrin, M. Y.: Large fluctuations of tropopause moisture over the Maritime Continent induced by a Kelvin wave during the SCOUT-O3 campaign in Darwin, Australia, Geophys. Res. Abstr., 9, 08845, 2007.

Canziani, P. O., Holton, J. R., Fishbein, E., Froidevaux, L., and Waters, J. W.: Equatorial Kelvin waves: A UARS MLS view, J. Atmos. Sci., 51, 3053-3076, 1994.

Canziani, P. O.: Slow and ultraslow equatorial Kelvin waves: The UARS CLAES view, Q. J. Roy. Meteor. Soc., 125, 657-676, doi:10.1256/smsqj.55413, 1999.

Chang, C.-P.: Vertical structures of tropical waves maintained by internally-induced cumulus heating, J. Atmos. Sci., 33, 729-739, 1976.

Cho, H.-K., Bowman, K. P., and North, G. R.: Equatorial waves including the Madden-Julian Oscillation in TRMM rainfall and OLR data, J. Climate, 17, 4387-4406, 2004.

de la Torre, A., Schmidt, T., and Wickert, J.: A global analysis of wave potential energy in the lower stratosphere derived from 5 years of GPS radio occultation data with CHAMP, Geophys. Res. Lett., 33, L24809, doi:10.1029/2006GL027696, 2006.

Dunkerton, T. J.: The role of gravity waves in the quasi-biennial oscillation, J. Geophys. Res., 102, 26 053-26 076, 1997.

Eckermann, S. D. and Preusse, P.: Global measurements of stratospheric mountain waves from space, Science, 286, 1534-1537, 1999.

Ern, M., Preusse, P., Alexander, M. J., and Warner, C. D.: Absolute values of gravity wave momentum flux derived from satellite data, J. Geophys. Res., 109, D20103, doi:10.1029/2004JD004752, 2004.

Ern, M., Preusse, P., and Warner, C. D.: A comparison between CRISTA satellite data and Warner and McIntyre gravity wave parameterization scheme: Horizontal and vertical wavelength filtering of gravity wave momentum flux, Adv. Space Res., 35, 2017 2023, doi:10.1016/j.asr.2005.04.109, 2005.

Ern, M., Preusse, P., and Warner, C. D.: Some experimental constraints for spectral parameters used in the Warner and McIntyre gravity wave parameterization scheme, Atmos. Chem. Phys., 6, 4361-4381, 2006, http://www.atmos-chem-phys.net/6/4361/2006/.

Fetzer, E. J. and Gille, J. C.: Gravity wave variances in LIMS temperatures, I, Variability and comparison with background winds, J. Atmos. Sci., 51, 2461-2483, 1994.

Fritts, D. C. and Alexander, M. J.: Gravity wave dynamics and effects in the middle atmosphere, Rev. Geophys., 41(1), 1003, doi:10.1029/2001RG000106, 2003.

Fulton, S. R. and Schubert, W. H.: Vertical normal mode transforms: Theory and application, Mon. Weather Rev., 113, 647658,1985

Garcia, R. R., Lieberman, R., Russell III, J. M., and Mlynczak, M. G.: Large-scale waves in the mesosphere and lower thermosphere observed by SABER, J. Atmos. Sci., 62, 4384-4399, 2005.

Hayashi, Y.: A method of estimating space-time spectra from polar- 
orbiting satellite data, J. Atmos. Sci., 37, 1385-1392, 1980.

Hitchman, M. H. and Leovy, C. B.: Estimation of the Kelvin wave contribution to the semiannual oscillation, J. Atmos. Sci., 45, 1462-1475, 1988.

Holton, J. R. and Lindzen, R. S.: An updated theory for the quasibiennial cycle of the tropical stratosphere, J. Atmos. Sci., 29, 1076-1080, 1972.

Johnson, R. H. and Ciesielski, P. E.: Rainfall and radiative heating rates from TOGA COARE atmospheric budgets, J. Atmos. Sci., 57, 1497-1514, 2000.

Jung, T. and Leutbecher, M.: Performance of the ECMWF forecasting system in the Arctic during winter, Q. J. Roy. Meteor. Soc., 133, 1327-1340, doi:10.1002/qj.99, 2007.

Krebsbach, M. and Preusse, P.: Spectral analysis of gravity wave activity in SABER temperature data, Geophys. Res. Lett., 34, L03814, doi:10.1029/2006GL028040, 2007.

Li, J., Wolf, W. W., Menzel, W. P., Zhang, W., Huang, H.-L., and Achtor, T. H.: Global soundings of the atmosphere from ATOVS measurements: The algorithm and validation, J. Appl. Meteorol., 39, 1248-1268, 2000.

Lieberman, R. S. and Riggin, D.: High resolution Doppler imager observations of Kelvin waves in the equatorial mesosphere and lower thermosphere, J. Geophys. Res., 102, 26 117-26 130, 1997.

Lindzen, R. S.: The interaction of waves and convection in the tropics, J. Atmos. Sci., 60, 3009-3020, 2003.

Marks, C. J. and Eckermann, S. D.: A three-dimensional nonhydrostatic ray-tracing model for gravity waves: Formulation and preliminary results for the middle atmosphere, J. Atmos. Sci., 52, 1959-1984, 1995.

Maruyama, T.: Upward transport of westerly momentum due to disturbances of the equatorial lower stratosphere in the period range of about 2 days - a Singapore data analysis for 1983-1993, J. Meteorol. Soc. Jpn., 72, 423-432, 1994.

Matsuno, T.: Quasi-geostrophic motions in the equatorial area, J. Meteorol. Soc. Jpn., 44, 25-43, 1966.

Mlynczak, M. G.: Energetics of the mesosphere and lower thermosphere and the SABER instrument, Adv. Space Res., 44, 11771183, 1997.

Moulin, F. Y. and Flór, J.-B.: Experimental study on wave breaking and mixing properties in the periphery of an intense vortex, Dynam. Atmos. Oceans, 40, 115-130, 2005

Olbers, D. J.: The propagation of internal waves in a geostrophic current, J. Phys. Oceanogr., 11, 1224-1233, 1981.

Pires, P., Redelsperger, J.-L., and Lafore, J.-P.: Equatorial atmospheric waves and their association to convection, Mon. Weather Rev., 125, 1167-1184, 1997.

Preusse, P., Eckermann, S. D., and Offermann, D.: Comparison of global distributions of zonal-mean gravity wave variance inferred from different satellite instruments, Geophys. Res. Lett., 27, 3877-3880, 2000.

Preusse, P., Dörnbrack, A., Eckermann, S. D., Riese, M., Schaeler, B., Bacmeister, J. T., Broutman, D., and Grossmann, K. U.: Space based measurements of stratospheric mountain waves by CRISTA, 1. Sensitivity, analysis method and a case study, J. Geophys. Res., 107, 8178, doi:10.1029/2001JD000699, 2002.

Preusse, P., Ern, M., Eckermann, S. D., Warner, C. D., Picard, R. H., Knieling, P., Krebsbach, M., Russell III, J. M., Mlynczak, M. G., Mertens, C. J., and Riese, M.: Tropopause to mesopause gravity waves in August: Measurement and modeling, J. Atmos. Sol.-Terr. Phys., 68, 1730-1751, 2006.

Randel, W. J., Boville, B. A., and Gille, J. C.: Observations of planetary mixed Rossby-gravity waves in the upper stratosphere, J. Atmos. Sci., 47, 3092-3099, 1990.

Randel, W. J. and Gille, J. C.: Kelvin wave activity in the upper stratosphere observed in SBUV ozone data, J. Atmos. Sci., 48, 2336-2349, 1991.

Randel, W. J. and Wu, F.: Kelvin wave variability near the equatorial tropopause observed in GPS radio occultation measurements, J. Geophys. Res., 110, D03102, doi:10.1029/2004JD005006, 2005.

Ratnam, M. V., Tsuda, T., Kozu, T., and Mori, S.: Long-term behavior of the Kelvin waves revealed by CHAMP/GPS RO measurements and their effects on the tropopause structure, Ann. Geophys., 24, 1355-1366, 2006, http://www.ann-geophys.net/24/1355/2006/.

Russell III, J. M., Mlynczak, M. G., Gordley, L. L., Tansock, J., and Esplin, R.: An overview of the SABER experiment and preliminary calibration results, Proceedings of SPIE, 3756, 277-288, 1999.

Salby, M. L.: Sampling theory for asynoptic satellite observations, Part I: Space-time spectra, resolution, and aliasing, J. Atmos. Sci., 39, 2577-2600, 1982.

Salby, M. L.: Sampling theory for asynoptic satellite observations, Part II: Fast Fourier synoptic mapping, J. Atmos. Sci., 39, 26012614, 1982.

Salby, M. L., Hartmann, D. L., Bailey, P. L., and Gille, J. C.: Evidence for equatorial Kelvin modes in Nimbus-7 LIMS, J. Atmos. Sci., 41, 220-235, 1984.

Salby, M. L. and Garcia, R. R.: Transient response to localized episodic heating in the tropics, Part I: Excitation and short-time near-field behavior, J. Atmos. Sci., 44, 458-498, 1987.

Salby, M. L., Matrosova, L., and Callaghan, P. F.: Global Kelvin waves in the upper atmosphere excited by tropospheric forcing at midlatitudes, J. Geophys. Res., 112, D06111, doi:10.1029/2006JD007235, 2007.

Sato, K., Hasegawa, F., and Hirota, I.: Short-period disturbances in the equatorial lower stratosphere, J. Meteorol. Soc. Jpn., 72, 859-873, 1994.

Sato, K. and Dunkerton, T. J.: Estimates of momentum flux associated with equatorial Kelvin and gravity waves, J. Geophys. Res., 102(D22), 26 247-26 261, 1997.

Semeniuk, K. and Shepherd, T. G.: Mechanisms for tropical upwelling in the stratosphere, J. Atmos. Sci., 58, 3097-3115, 2001.

Shiotani, M., Gille, J. C., and Roche, A. E.: Kelvin waves in the equatorial lower stratosphere as revealed by cryogenic limb etalon spectrometer temperature data, J. Geophys. Res., 102(D22), 26 131-26 140, 1997.

Shuckburgh, E., Norton, W., Iwi, A., and Haynes, P.: Influence of the quasi-biennial oscillation on isentropic transport and mixing in the tropics and subtropics, J. Geophys. Res., 106(D13), 14327-14 337, 2001

Smith, A. K., Preusse, P., and Oberheide, J.: Middle atmosphere Kelvin waves observed in Cryogenic Infrared Spectrometers and Telescopes for the Atmosphere (CRISTA) 1 and 2 temperature and trace species, J. Geophys. Res., 107(D23), 8177, doi:10.1029/2001JD000577, 2002.

Srikanth, R. and Ortland, D. A.: Analysis of Kelvin waves in High- 
Resolution Doppler Imager and Microwave Limb Sounder stratosphere measurements using a constrained least squares method, J. Geophys. Res., 103(D18), 23 131-23 151, 1998.

Straub, K. H. and Kiladis, G. N.: The observed structure of convectively coupled Kelvin waves: Comparison with simple models of coupled wave instability, J. Atmos. Sci., 60, 1655-1668, 2003.

Takahashi, H., Wrasse, C. M., Fechine, J., Pancheva, D., Abdu, M. A., Batista, I. S., Lima, L. M., Batista, P. P., Clemesha, B. R., Schuch, N. J., Shiokawa, K., Gobbi, D., Mlynczak, M. G., and Russell, J. M.: Signature of ultra fast Kelvin waves in the equatorial middle atmosphere and ionosphere, Geophys. Res. Lett., 34, L11108, doi:10.1029/2007GL029612, 2007.

Tindall, J. C., Thuburn, J., and Highwood, E. J.: Equatorial waves in the lower stratosphere. I: A novel detection method, Q. J. Roy. Meteor. Soc., 132, 177-194, doi:10.1256/qj.04.152, 2006.

Tindall, J. C., Thuburn, J., and Highwood, E. J.: Equatorial waves in the lower stratosphere. II: Annual and interannual variability, Q. J. Roy. Meteor. Soc., 132, 195-212, doi:10.1256/qj.04.153, 2006.

Tsai, H.-F., Tsuda, T., Hajj, G. A., Wickert, J., and Aoyama, Y.: Equatorial Kelvin waves observed with GPS occultation measurements (CHAMP and SAC-C), J. Meteorol. Soc. Jpn., 82, 397-406, 2004.

Vincent, R. A. and Alexander, M. J.: Gravity waves in the tropical lower stratosphere: An observational study of seasonal and interannual variability, J. Geophys. Res., 105(D14), 17971-17982, 2000.
Wallace, M. W. and Kousky, V. E.: Observational evidence of Kelvin waves in the tropical stratosphere, J. Atmos. Sci., 25, 900-907, 1968.

Wheeler, M. and Kiladis, G. N.: Convectively coupled equatorial waves: Analysis of clouds and temperature in the wavenumberfrequency domain, J. Atmos. Sci., 56, 374-399, 1999.

Wu, D. L., Hays, P. B., and Skinner, W. R.: A least squares method for spectral analysis of space-time series, J. Atmos. Sci., 52, 3501-3511, 1995.

Wu, D. L., Preusse, P., Eckermann, S. D., Jiang, J. H., Juarez, M. D. L. T., Coy, L., and Wang, D. Y.: Remote sounding of atmospheric gravity waves with satellite limb and nadir techniques, Adv. Space Res., 37, 2269-2277, 2006.

Wu, D. L.: Small-scale fluctuations and scintillations in highresolution GPS/CHAMP SNR and phase data, J. Atmos. Sol.Terr. Phys., 68, 999-1017, 2006.

Wu, Z., Sarachik, E. S., and Battisti, D. S.: Vertical structure of convective heating and the three-dimensional structure of the forced circulation on an equatorial beta plane, J. Atmos. Sci., 57, 21692187, 2000.

Yanai, M. and Maruyama, T.: Stratospheric wave disturbances propagating over the equatorial Pacific, J. Meteorol. Soc. Jpn., 44, 291-294, 1966.

Yee, J. H., Talaat, E. R., Christensen, A. B., Killeen, T. L., Russell, J. M., and Woods, T. N.: TIMED instruments, Johns Hopkins APL Technical Digest, 24, 156-164, 2003. 